\title{
Unifying the Galilei Relativity and the Special Relativity
}

\author{
Alexandre Lyra' ${ }^{1}$ and Marcelo Carvalho ${ }^{2}$ \\ ${ }^{1}$ Universidade Federal do Rio de Janeiro, Observatório do Valongo, 20080-090 Rio de Janeiro, RJ, Brazil \\ ${ }^{2}$ Departamento de Matemática, Universidade Federal de Santa Catarina, 88.040-900 Florianópolis, SC, Brazil
}

Correspondence should be addressed to Marcelo Carvalho; marcelo_carv@hotmail.com

Received 4 April 2013; Accepted 8 May 2013

Academic Editors: G. Cleaver, A. Herrera-Aguilar, D. Singleton, and F. Sugino

Copyright (C) 2013 A. Lyra and M. Carvalho. This is an open access article distributed under the Creative Commons Attribution License, which permits unrestricted use, distribution, and reproduction in any medium, provided the original work is properly cited.

We present two models combining some aspects of the Galilei and the Special relativities that lead to a unification of both relativities. This unification is founded on a reinterpretation of the absolute time of the Galilei relativity that is considered as a quantity in its own and not as mere reinterpretation of the time of the Special relativity in the limit of low velocity. In the first model, the Galilei relativity plays a prominent role in the sense that the basic kinematical laws of Special relativity, for example, the Lorentz transformation and the velocity law, follow from the corresponding Galilei transformations for the position and velocity. This first model also provides a new way of conceiving the nature of relativistic spacetime where the Lorentz transformation is induced by the Galilei transformation through an embedding of 3-dimensional Euclidean space into hyperplanes of 4-dimensional Euclidean space. This idea provides the starting point for the development of a second model that leads to a generalization of the Lorentz transformation, which includes, as particular cases, the standard Lorentz transformation and transformations that apply to the case of superluminal frames.

\section{Introduction}

It is common to consider the Galilei relativity as the low velocity limit case of the Special relativity (SR). This is revealed by the behavior of the Lorentz transformation when we take $v / c \rightarrow 0$; for example,

$$
\begin{gathered}
\vec{x}^{\prime}=\vec{x}-(1-\gamma) \frac{\vec{x} \cdot \vec{v}}{v^{2}} \vec{v}-\gamma \vec{v} t \longrightarrow \vec{x}^{\prime}=\vec{x}-\vec{v} t, \\
t^{\prime}=\gamma\left(t-\frac{\vec{x} \cdot \vec{v}}{c^{2}}\right) \longrightarrow t^{\prime}=t .
\end{gathered}
$$

In this view, the absolute time of the Galilei relativity, henceforth denoted by $\tau$, is not considered as an independent quantity but it refers to a particular situation of SR when it is possible to identify $t$ and $t^{\prime}$, which allows us to take $\tau \equiv t=t^{\prime}$. It is in this sense that the absolute time is usually conceived. Therefore, the fact that one generally has $t^{\prime} \neq t$ seems to deny the possibility of having an absolute time in SR. We refer to the time of the SR as the physical time.

In our work we intend to reinterpret the notion of absolute time in such a way that the Galilei relativity recovers its role as a theory in its own, correcting then a common view that treats the principles of the Galilei and the Special relativities as irreconcilable notions. One of the difficulties we encounter to carry out this goal is related to the way we currently understand the concept of time, which is somehow already shaped by ideas of the SR. Here, we focus our efforts on the development of two models, called models I and II, that are built in order to allow to introduce the concept of absolute time in a general setup that also incorporates the concept of time of SR.

In model $\mathrm{I}$, the fact that $t \neq t^{\prime}$ suggest us not to identify the absolute time with $t$ but with a function depending on $t$ and, perhaps, on the spatial location where the event occurred. Then, if for a certain instant $\tau$ we have $(t, \vec{x}),\left(t^{\prime}, \vec{x}^{\prime}\right)$ representing the same event with respect to two inertial frames, we would expect the absolute character of $\tau$ to imply that $\tau(t, \vec{x})=$ $\tau\left(t^{\prime}, \vec{x}^{\prime}\right)$, with this expression being verified for any value of the ratio $v / c$. In addition, we would also expect to obtain the Lorentz transformation by replacing $\tau(t, \vec{x})$ into the Galilei law $\vec{x}^{\prime}=\vec{x}-\vec{v} \tau$. Having established this, the next step would be to obtain the velocity law of SR from the corresponding velocity law of the Galilei relativity, $d \vec{x}^{\prime} / d \tau=(d \vec{x} / d \tau)-\vec{v}$. 
The fulfillment of these two laws places the Galilei relativity as a guiding principle for deriving some of the SR laws.

From a calculational perspective, the Lorentz transformation can be obtained by considering as variables the set $\left\{t, \vec{x}, t^{\prime}, \vec{x}^{\prime}\right\}$, together with the equation $\vec{x}^{\prime 2}-c^{2} t^{\prime 2}=\vec{x}^{2}-$ $c^{2} t^{2}$. In model I, we will show that in order to combine the Galilei and the Special relativities, it is sufficient to enlarge the variables' set of SR to $\left\{t, \vec{x}, t^{\prime}, \vec{x}^{\prime}, \tau\right\}$ and to consider the pair of equations

$$
\begin{gathered}
\vec{x}^{\prime 2}-c^{2} t^{\prime 2}=\vec{x}^{2}-c^{2} t^{2}, \\
\vec{x}^{\prime}=\vec{x}-\vec{v} \tau .
\end{gathered}
$$

Here, the effect of this enlargement is to produce not only the usual Lorentz transformation, but also an extra equation relating the absolute and the physical time, which gives an operational definition for the absolute time associated to the occurrence of an event.

In what concerns the structure of spacetime, we notice that both relativities are formulated in terms of a 4dimensional space $\mathbb{R}^{4} \equiv \mathbb{R} \times \mathbb{R}^{3}$. In the Galilei view, two observers belonging to inertial frames $S, S^{\prime}$ describe the occurrence of an event, respectively, as a point $(\tau, \vec{x}) \in \mathbb{R}_{S} \times$ $\mathbb{R}_{S}^{3} \equiv \mathbb{R}_{S}^{4}$ and $\left(\tau, \vec{x}^{\prime}\right) \in \mathbb{R}_{S^{\prime}} \times \mathbb{R}_{S^{\prime}}^{3} \equiv \mathbb{R}_{S^{\prime}}^{4}$. Here, due to the nature of the absolute time, for a fixed $\tau$, the Galilei transformation reduces itself to a map $\mathbb{R}_{S}^{3} \rightarrow \mathbb{R}_{S^{\prime}}^{3}$.

In the SR view, the observers describe the event as a point $(t, \vec{x}) \in \mathbb{R}_{S} \times \mathbb{R}_{S}^{3}$, and $\left(t^{\prime}, \vec{x}^{\prime}\right) \in \mathbb{R}_{S^{\prime}} \times \mathbb{R}_{S^{\prime}}^{3}$ where now, due to the nature of the physical time, the Lorentz transformation is not restricted to be a map $\mathbb{R}_{S}^{3} \rightarrow \mathbb{R}_{S^{\prime}}^{3}$ as before, but it is considered as a map on the whole space, $\mathbb{R}_{S}^{4} \rightarrow \mathbb{R}_{S^{\prime}}^{4}$. In model I, the existence of the absolute time allows us to unveil an additional structure present in the SR spacetime that is already present in the Galilei view. In fact, when we express the absolute time in terms of the physical time, $\tau(t), \tau\left(t^{\prime}\right)$, we will show that we can define embeddings $\mathbb{R}_{S}^{3} \stackrel{i_{\tau}}{\hookrightarrow} \mathbb{R}_{S}^{4}$, $\mathbb{R}_{S^{\prime}}^{3} \stackrel{i_{\tau}^{\prime}}{\hookrightarrow} \mathbb{R}_{S^{\prime}}^{4}$ in terms of certain hyperplanes $\sigma_{\tau} \subset \mathbb{R}_{S}^{4}, \sigma_{\tau}^{\prime} \subset \mathbb{R}_{S^{\prime}}^{4}$. The Lorentz transformation is then seen as a map $\sigma_{\tau} \rightarrow \sigma_{\tau}^{\prime}$ between these 3-dimensional hyperplanes that is induced by the Galilei transformation acting on $\mathbb{R}_{S}^{3} \rightarrow \mathbb{R}_{S^{\prime}}^{3}$, for a certain $\tau$. Therefore, we may see the spacetime as the union of these hyperplanes, for example, $\mathbb{R}_{S}^{4}=\cup_{\tau} \sigma_{\tau}$. In the spacetime of Galilei relativity this splitting is self-evident and corresponds to the hyperplanes of $\tau=$ constant.

Inspired by the equation that defines the embeddings $\mathbb{R}_{S}^{3} \stackrel{i_{\tau}}{\hookrightarrow} \mathbb{R}_{S}^{4}, \mathbb{R}_{S^{\prime}}^{3} \stackrel{i_{\tau}^{\prime}}{\hookrightarrow} \mathbb{R}_{S^{\prime}}^{4}$, we develop model II by taking $\alpha x^{0}-\vec{\beta} \cdot \vec{x}=\alpha x^{\prime 0}+\vec{\beta} \cdot \vec{x}^{\prime}$ as our fundamental equation, where $\alpha, \vec{\beta}$ are arbitrary parameters that will allow us to define a generalization of the Lorentz transformation. In model II, the absolute time is introduced by modifying the previous equation to

$$
\tau \equiv \alpha x^{0}-\vec{\beta} \cdot \vec{x}=\alpha x^{\prime 0}+\vec{\beta} \cdot \vec{x}^{\prime} .
$$

Here, the Galilei relativity arises assuming in addition the relation $\vec{x}^{\prime}=\vec{x}-\vec{v} \tau$. This is sufficient to reduce the generalized transformation to the particular form of the standard
Lorentz transformation, showing then the consistency of both relativities. In model II, however, we will show that we are allowed to have the Lorentz transformation weather or not we consider the Galilei relativity, a situation that does not happen in model $\mathrm{I}$.

Some works $[1,2]$ deal with the Galilei and the Special relativities but focus on opposite goals not proposing a scheme for unifying both relativities. In fact, in [1] the assumption that the Galilei law of velocity applies to the motion of bodies, signals, and forces leads to inconsistencies that are solved by the introduction of the postulate of the constancy of the speed of light, which ultimately leads to SR. In [2], a transformation relating the Galilei and the Minkowski-Einstein coordinates is established, which may signalize that relativistic effects are due to motion relative to an actual 3-space. (The author continues this development in a model that is known by Process Physics.) In our work, we follow another direction as our goal is to harmonize both relativities by a convenient treatment of the absolute time and the fundamentals laws of both relativities. We are not aware of any work devoted to this issue; therefore, we hope that our work may provide a convenient starting point for further investigation on this topic.

Our work is organized as follows. Model I is developed in Section 3. In Section 3.1 we introduce a set of assumptions settling the main properties of the physical space and time that are necessary to develop a model incorporating both the Galilei and the Special relativities. We axiomatize the existence of two times, the absolute time of the Galilei relativity and the physical time of SR, and we discuss an important issue, due to Møller, concerning the correct interpretation of the physical space where (1) is defined. In Section 3.2 we obtain the Lorentz transformation from the Galilei law $\vec{x}^{\prime}=\vec{x}-\vec{v} \tau$ and the assumptions of Section 3.1. In Section 3.4 we obtain from the law of velocity transformation of Galilei relativity the corresponding velocity law of SR. We also discuss a problem associated to the so-called Thomas precession. In Section 3.5 we give a geometric interpretation for the spacetime of SR and show how the concept of absolute time allows to define a partition of the spacetime in terms of certain 3-dimensional hyperplanes. Model II is developed in Section 4. In Section 4.1 we establish a set of assumptions that is slightly different than the assumptions of model I, namely, in what concerns the introduction of the Special and the Galilei relativities (resp., numbered as assumptions (III) and (IV)). In Section 4.2 we derive a transformation that we call Generalized Lorentz Transformation, and in Section 4.3 we analyze the velocity transformation associated to it. We then show that the Generalized Lorentz Transformation includes, as particular cases, the standard Lorentz transformation together with transformations that may be used when we have superluminal frames. We then analyze in Section 4.4 how to introduce the Galilei relativity in the framework of model II. In Section 4.5 we analyze the conservation of momentum and obtain the corresponding relativistic expression for the mass. Finally, in Section 5 we analyze how elementary considerations from the differential structure of the projective space $\mathbb{R P}(5)$ allow us to think on the concept of the absolute time as associated to one of the dimensions of Euclidean space $\mathbb{R}^{4}$. 


\section{Basic Definitions}

We recall some basic definitions that are necessary for the statement of our assumptions.

An event is any physical occurrence taking place on a certain location and in a certain instant. We adopt the standard definitions of observer and reference frame as stated concisely in [3]; that is, by an observer we understand any entity equipped with a standard rod and a standard clock that allows for the measurement of length and time (in fact, the physical time as we will introduce in the assumption (II)) and that is able to communicate with other observers by means of light signals. A reference frame is understood as an infinite set of observers each one at rest relative to the other and having their standard clocks synchronized. Abstractly, the infinite set of observers composing a reference frame is idealized in such a way that for any event there is an observer present on the same location where the event took place, which then establishes the space coordinate of the event. This observer also determines, by the reading of his clock, the instant of time when the event occurred. We will consider reference frames endowed with a rectangular coordinate system. By an inertial frame we understand any frame in which a body free of forces is unaccelerated.

We use the notation $\left(t_{S P}, \vec{x}_{S P}\right)$ to denote the description of an event $P$ relative to a frame $S$. We write $\left(t_{S P}, \vec{x}_{S P}\right) \sim$ $\left(t_{S^{\prime} P}, \vec{x}_{S^{\prime} P}\right)$ to indicate the same event $P$ as seen by the frames $S, S^{\prime}$. In the particular case when one frame $S$ analyzes the movement of another frame $S^{\prime}$, we will assume that the frame $S^{\prime}$ is entirely represented by its origin and write $\vec{x}_{S S^{\prime}}$ for the position of $S^{\prime}$ relative to $S$, and $t_{S S^{\prime}}$ for the time measured by $S$. Finally, in our work by a Lorentz transformation we always mean a Lorentz boost, except in Section 5 where a Lorentz boost attains its precise meaning as a particular Lorentz transformation.

\section{Model I}

3.1. The Assumptions. In what concerns space and time, our basic assumptions are as follows.

(I) Space. Each inertial frame describes space as being an euclidean 3-dimensional vector space.

(II) Time. We model time as any variable that can be used to describe what we intuitively understand as the "instant when events occur." We distinguish two kinds of choices as follows. By physical time, denoted by $t$, we understand a choice for the time variable that can be measured with the use of standard devices, like atomic clocks, under suitable arrangements (e.g., when they are the clocks of a reference frame and are all conveniently synchronized, etc.). By absolute time, denoted by $\tau$, we understand a choice for the time variable having the property that given an event all observers assign to it the same value for the instant when the event occurred. (Later, through the analysis of their transformation properties, we will identify the physical time as the ordinary time of the special relativity, while the absolute time will be identified with the time of the Galilei relativity.) As a principle, we assume that any variable that serves to describe time may be expressed in terms of the physical time in such way that by the measurement of the latter one can determine the value of the former. Therefore, for any frame in which one knows how to shift from $\tau(t) \leftrightarrow t(\tau)$ it is possible to describe an event writing its coordinates as $(t, \vec{x})$ or $(\tau, \vec{x})$.

(III) Given two inertial frames $S, S^{\prime}$ moving with relative velocity $\vec{v}$ and an event $P$ whose coordinates are $\left(t_{S P}, \vec{x}_{S P}\right),\left(t_{S^{\prime} P}, \vec{x}_{S^{\prime} P}\right)$ relative to $S, S^{\prime}$, we have

$$
\vec{x}_{S P}^{2}-c^{2} t_{S P}^{2}=\vec{x}_{S^{\prime} P}^{2}-c^{2} t_{S^{\prime} P}^{2} .
$$

When we consider speed calculated in terms of derivatives relative to the physical time, the relation (4) expresses the constancy of the speed of light.

(IV) The Galilean Relativity Principle. Given two inertial frames $S, S^{\prime}$ moving with relative velocity $\vec{v}$ and an event $P$ whose coordinates are $\left(\tau, \vec{x}_{S P}\right),\left(\tau, \vec{x}_{S^{\prime} P}\right)$ relative to $S, S^{\prime}$, we have componentwise that

$$
\vec{x}_{S^{\prime} P}=\vec{x}_{S P}-\vec{v}_{S S^{\prime}} \tau \text {. }
$$

(V) The relation between $\left(t_{S P}, \vec{x}_{S P}\right)$ and $\left(t_{S^{\prime} P}, \vec{x}_{S^{\prime} P}\right)$ is linear.

Remarks. (i) In assumption (I), it is assumed that observers in inertial frames see physical space endowed with an euclidean structure. This assumption is supported by the lack of evidence signalyzing deviations of space properties from the euclidean structure (see the discussion of French in [4, pages 59-61]). The position of an event $P$ relative to inertial frames $S, S^{\prime}$ is written as $\vec{x}_{S P} \equiv\left(x^{1}, x^{2}, x^{3}\right) \in$ $\mathbb{R}_{S}^{3}, \vec{x}_{S^{\prime} P} \equiv\left(x^{\prime 1}, x^{\prime 2}, x^{\prime 3}\right) \in \mathbb{R}_{S^{\prime}}^{3}$ and since each frame is endowed with its own coordinate system, the spaces $\mathbb{R}_{S}^{3}$ and $\mathbb{R}_{S^{\prime}}^{3}$ are conceived as distinct spaces; therefore, we must understand the relation between the vectors $\vec{x}_{S P}$ and $\vec{x}_{S^{\prime} P}$ established in (5) not as a vector equation defined in one and the same vector space. However, it is possible to do so if we reinterpret the vectors $\vec{x}_{S P}$ and $\vec{x}_{S^{\prime} P}$ as follows. (This reasoning is originally due to Møller [5].) Given two frames $S, S^{\prime}$ we assume that there is a single space $\mathbb{R}_{S S^{\prime}}^{3}$ where the components $\left(x^{1}, x^{2}, x^{3}\right),\left(x^{\prime 1}, x^{\prime 2}, x^{\prime 3}\right)$ of the vectors $\vec{x}_{S P}, \vec{x}_{S^{\prime} P}$ are seen as images of maps

$$
\begin{aligned}
\mathbb{R}_{S}^{3} \longrightarrow \mathbb{R}_{S S^{\prime}}^{3}: \vec{x}_{S P} \longrightarrow\left(x^{1}, x^{2}, x^{3}\right) \\
\mathbb{R}_{S^{\prime}}^{3} \longrightarrow \mathbb{R}_{S S^{\prime}}^{3}: \vec{x}_{S^{\prime} P} \longrightarrow\left(x^{\prime 1}, x^{\prime 2}, x^{\prime 3}\right)
\end{aligned}
$$

and such that for a fixed $\tau$ they satisfy (5); that is, we can think of the vectors $\vec{x}_{S P}, \vec{x}_{S^{\prime} P}$ as vectors in $\mathbb{R}_{S S^{\prime}}^{3}$, which justify using the same notation for them. Equation (5) is now understood as an equation defined in $\mathbb{R}_{S S^{\prime}}^{3}$.

From the perspective of the Galilei relativity, it is clear that the vector $\vec{x}_{S^{\prime} P} \in \mathbb{R}_{S^{\prime}}^{3}$ can be measured directly from the observers of the frame $S$ that at the instant $\tau$ are placed, 
respectively, at the positions corresponding to the origin of the frame $S^{\prime}$ and the event $P$; that is, componentwise we identify $\mathbb{R}_{S^{\prime}}^{3} \ni \vec{x}_{S^{\prime} P} \leftrightarrow \vec{\eta} \in \mathbb{R}_{S}^{3}$,

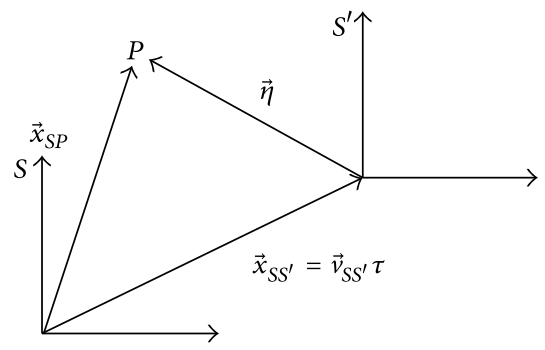

where

$$
\vec{\eta}:=\vec{x}_{S P}-\vec{x}_{S S^{\prime}}=\vec{x}_{S P}-\vec{v}_{S S^{\prime}} \tau
$$

This corresponds to the same equation (5), now referred entirely to the space $\mathbb{R}_{S}^{3}$. This procedure is equivalent to identify $\mathbb{R}_{S S^{\prime}}^{3}$ with $\mathbb{R}_{S}^{3}$. A similar procedure allows to identify $\mathbb{R}_{S S^{\prime}}^{3}$ and $\mathbb{R}_{S^{\prime}}^{3}$. The assumption that spaces $\mathbb{R}_{S}^{3}, \mathbb{R}_{S^{\prime}}^{3}$ are euclidean is then consistent with the interpretation we obtain for (5) through the identification $\mathbb{R}_{S}^{3} \simeq \mathbb{R}_{S S^{\prime}}^{3}, \mathbb{R}_{S^{\prime}}^{3} \simeq \mathbb{R}_{S S^{\prime}}^{3}$.

A different picture emerges in SR. Now, instead of (5) we have the relation (it seems it was Møller (see [5, Section 2.4]) who first noticed the importance of giving a correct interpretation for (9) as an equation established in an abstract vector space, where it would make sense to relate to the vectors $\vec{x}_{S P}$ and $\vec{x}_{S^{\prime} P}$ that, in principle, belong to different spaces. Møller did so thinking exclusively from the perspective of the SR. However, the same question is already manifest from the perspective of the Galilei relativity if we intend to interpret correctly (5))

$$
\vec{x}_{S^{\prime} P}=\vec{x}_{S P}-(1-\gamma) \frac{\vec{x}_{S P} \cdot \vec{v}_{S S^{\prime}}}{v_{S S^{\prime}}^{2}} \vec{v}_{S S^{\prime}}-\gamma \vec{v}_{S S^{\prime}} t_{S P} .
$$

Here, the vectors $\vec{x}_{S P} \in \mathbb{R}_{S}^{3}, \vec{x}_{S^{\prime} P} \in \mathbb{R}_{S^{\prime}}^{3}$ are mapped into an abstract space $V_{S S^{\prime}}$, where we understand that (9) is defined. From the perspective of SR and according to the frame $S$, if the event $P$ occurs at the instant $t_{S P}$, the observers on the frame $S$ that occupy, respectively, the positions of the origin of the frame $S^{\prime}$ and the event $P$ allow us to define a vector $\vec{\eta}$

$$
\vec{\eta}:=\vec{x}_{S P}-\vec{v}_{S S^{\prime}} t_{S P}
$$

which is not componentwise equal to the vector $\vec{x}_{S^{\prime} P}$ as measured by the frame $S^{\prime}$

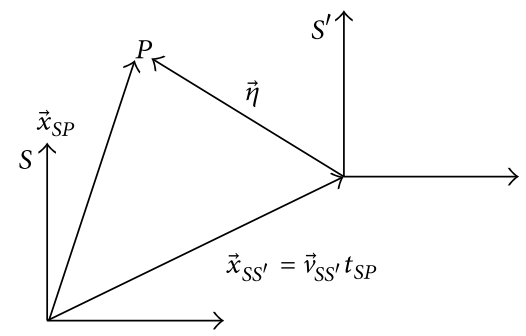

In fact, using the relation

$$
t_{S^{\prime} P}=\gamma\left(t_{S P}-\frac{\vec{x}_{S P} \cdot \vec{v}_{S S^{\prime}}}{c^{2}}\right)
$$

together with (9) to write $\vec{x}_{S P}$ in terms of $\vec{x}_{S^{\prime} P}, t_{S^{\prime} P}$, we obtain componentwise that

$$
\vec{\eta}=\vec{x}_{S^{\prime} P}+\frac{1-\gamma}{\gamma} \frac{\vec{x}_{S^{\prime} P} \cdot \vec{v}_{S^{\prime} S}}{v_{S^{\prime} S}^{2}} \vec{v}_{S^{\prime} S} .
$$

Therefore, from the perspective of SR, we cannot identify the vectors $\vec{\eta}$ and $\vec{x}_{S^{\prime} P}$ as we did before. Physically, this means that measurements performed by the observers of the frame $S$ are not sufficient to identify (10) with (9) of $V_{S S^{\prime}}$. We then distinguish the spaces $\mathbb{R}_{S S^{\prime}}^{3}$ and $V_{S S^{\prime}}$ in the sense that we cannot identify $\mathbb{R}_{S}^{3}$ or $\mathbb{R}_{S^{\prime}}^{3}$ with $V_{S S^{\prime}}$ as we did in the case of the Galilei relativity.

(ii) In assumption (III), we consider that (4) holds not only for the events associated with the movement of a light ray, but also for every event. As we know [6], in the standard treatment of SR if we assume that (4) is verified for a light ray, then from the assumption of the linearity of the transformation involving $\vec{x}, t$ and $\vec{x}^{\prime}, t^{\prime}$ we obtain that condition (4) is also verified for any event. In our approach, since we work essentially with two equations (4), (5) and an extended set of variables $\left\{\tau, t, t^{\prime}, \vec{x}, \vec{x}^{\prime}\right\}$, there is no guarantee that assuming that (4) is true for a light ray would imply its validity for all events. (For the case of the standard treatment of the SR, Landau and Lifshitz give a heuristic argument justifying it in [7, page 5], while Einstein put it axiomatically in [8, appendix one].) Therefore, in the development of our model it is necessary to assume from the beginning that (4) is verified for not only for those events associated to the movement of a light ray, but also all events. This will become evident in the derivation shown in Section 3.2.

(iii) The physical and the absolute time introduced in assumption (II) assume their specific characteristic from the conditions they have to obey in assumptions (III) and (IV). These conditions identify $t$ as the time of Special relativity and $\tau$ as the time of Galilei relativity.

(iv) Whenever we set a transformation between two frames we assume that at $\tau=0$, or equivalently at $t=t^{\prime}=0$, the origins of both frames coincide and their coordinate axes are parallel.

(v) In assumption (V), by a linear relation between the pairs $(t, \vec{x}),\left(t^{\prime}, \vec{x}^{\prime}\right)$ we mean that $t^{\prime}$ and $\vec{x}^{\prime}$ depend only on the first-order power of $t, \vec{x}$.

3.2. Deriving the Transformation. In order to obtain the transformations we start from assumption (IV)

$$
\vec{x}_{S^{\prime} P}=\vec{x}_{S P}-\vec{v}_{S S^{\prime}} \tau
$$

which gives

$$
\vec{x}_{S^{\prime} P}^{2}=\vec{x}_{S P}^{2}-2 \vec{x}_{S P} \cdot \vec{v}_{S S^{\prime}} \tau+v_{S S^{\prime}}^{2} \tau^{2} .
$$

From assumption (III) we obtain

$$
v_{S S^{\prime}}^{2} \tau^{2}-2 \vec{x}_{S P} \cdot \vec{v}_{S S^{\prime}} \tau+c^{2}\left(t_{S P}^{2}-t_{S^{\prime} P}^{2}\right)=0
$$


that allows us to write

$$
\tau=\frac{\vec{x}_{S P} \cdot \vec{v}_{S S^{\prime}} \pm \sqrt{\left(\vec{x}_{S P} \cdot \vec{v}_{S S^{\prime}}\right)^{2}-v_{S S^{\prime}}^{2} c^{2}\left(t_{S P}^{2}-t_{S^{\prime} P}^{2}\right)}}{v_{S S^{\prime}}^{2}} .
$$

Now, according to assumption (V), we look for a relation between $t_{S P}$ and $t_{S^{\prime} P}$ such that the corresponding expression for $\tau$ given in (17), when replaced into (14), results in a transformation involving at most terms to the first power in $\vec{x}_{S^{\prime} P}, \vec{x}_{S P}$. In order to obtain that, let us consider the following particular relation between $t_{S P}$ and $t_{S^{\prime} P}$, for example,

$$
t_{S^{\prime} P}=a t_{S P}+b \vec{x}_{S P} \cdot \vec{v}_{S S^{\prime}}
$$

which gives $t_{S P}^{2}-t_{S^{\prime} P}^{2}=\left(1-a^{2}\right) t_{S P}^{2}-2 a b \vec{x}_{S P} \cdot \vec{v}_{S S^{\prime}} t_{S P}-b^{2}\left(\vec{x}_{S P}\right.$. $\left.\vec{v}_{S S^{\prime}}\right)^{2}$. The constants $a$ and $b$ must be chosen in such way that $\left(\vec{x}_{S P} \cdot \vec{v}_{S S^{\prime}}\right)^{2}-v_{S S^{\prime}}^{2} c^{2}\left(t_{S P}^{2}-t_{S^{\prime} P}^{2}\right)$ becomes a perfect square, that is,

$$
\begin{aligned}
\left(\vec{x}_{S P}\right. & \left.\cdot \vec{v}_{S S^{\prime}}\right)^{2}-v_{S S^{\prime}}^{2} c^{2}\left(t_{S P}^{2}-t_{S^{\prime} P}^{2}\right) \\
= & \left(1+v_{S S^{\prime}}^{2} c^{2} b^{2}\right)\left(\vec{x}_{S P} \cdot \vec{v}_{S S^{\prime}}\right)^{2} \\
& +2 v_{S S^{\prime}}^{2} c^{2} a b \vec{x}_{S P} \cdot \vec{v}_{S S^{\prime}} t_{S P} \\
& +\left(a^{2}-1\right) v_{S S^{\prime}}^{2} c^{2} t_{S P}^{2} \\
\equiv & {\left[\left(\vec{x}_{S P} \cdot \vec{v}_{S S^{\prime}}\right) \sqrt{1+v_{S S^{\prime}}^{2} c^{2} b^{2}}-\sqrt{a^{2}-1} c v_{S S^{\prime}} t_{S P}\right]^{2} }
\end{aligned}
$$

which gives $a^{2}=1+v_{S S^{\prime}}^{2} c^{2} b^{2}$. (We could have written (19) in the form $\left(\vec{x}_{S P} \cdot \vec{v}_{S S^{\prime}}\right) \sqrt{1+v_{S S^{\prime}}^{2} c^{2} b^{2}}+\sqrt{a^{2}-1} c v_{S S^{\prime}} t_{S P}$. As we will see, the minus sign adopted in (19) becomes necessary if we intend to obtain the standard Lorentz transformation of SR.) We have then

$$
\tau=\frac{\vec{x}_{S P} \cdot \vec{v}_{S S^{\prime}} \pm\left|\vec{x}_{S P} \cdot \vec{v}_{S S^{\prime}}\right| a\left|-\sqrt{a^{2}-1} c v_{S S^{\prime}} t_{S P}\right|}{v_{S S^{\prime}}^{2}} .
$$

Here, the choice for the sign of $\tau$ is fixed as follows. Replacing $\tau$ given by (20) into (14) and considering the time transformation (18), we assume that the transformation $\left(t_{S P}, \vec{x}_{S P}\right) \rightarrow$ $\left(t_{S^{\prime} P}, \vec{x}_{S^{\prime} P}\right)$ is invertible upon replacing $\left(t_{S^{\prime} P}, \vec{x}_{S^{\prime} P}, \vec{v}_{S^{\prime} S}\right) \leftrightarrow$ $\left(t_{S P}, \vec{x}_{S P}, \vec{v}_{S S^{\prime}}\right)$ with $\vec{v}_{S^{\prime} S}=-\vec{v}_{S S^{\prime}}$. Therefore, we notice the following.

(i) If $\vec{x}_{S P} \cdot \vec{v}_{S S^{\prime}}|a|-\sqrt{a^{2}-1} c v_{S S^{\prime}} t_{S P} \geq 0$, we must choose

$$
\begin{aligned}
\tau & =\frac{\vec{x}_{S P} \cdot \vec{v}_{S S^{\prime}}-\left|\vec{x}_{S P} \cdot \vec{v}_{S S^{\prime}}\right| a\left|-\sqrt{a^{2}-1} c v_{S S^{\prime}} t_{S P}\right|}{v_{S S^{\prime}}^{2}} \\
& =(1-|a|) \frac{\vec{x}_{S P} \cdot \vec{v}_{S S^{\prime}}}{v_{S S^{\prime}}^{2}}+\frac{\sqrt{a^{2}-1}}{v_{S S^{\prime}}} c t_{S P} .
\end{aligned}
$$

(ii) If $\vec{x}_{S P} \cdot \vec{v}_{S S^{\prime}}|a|-\sqrt{a^{2}-1} c v_{S S^{\prime}} t_{S P}<0$, we must choose

$$
\begin{aligned}
\tau & =\frac{\vec{x}_{S P} \cdot \vec{v}_{S S^{\prime}}+\left|\vec{x}_{S P} \cdot \vec{v}_{S S^{\prime}}\right| a\left|-\sqrt{a^{2}-1} c v_{S S^{\prime}} t_{S P}\right|}{v_{S S^{\prime}}^{2}} \\
& =(1-|a|) \frac{\vec{x}_{S P} \cdot \vec{v}_{S S^{\prime}}}{v_{S S^{\prime}}^{2}}+\frac{\sqrt{a^{2}-1}}{v_{S S^{\prime}}} c t_{S P} .
\end{aligned}
$$

In both cases, we obtain the transformation

$$
\begin{gathered}
\vec{x}_{S^{\prime} P}=\vec{x}_{S P}-(1-|a|) \frac{\vec{x}_{S P} \cdot \vec{v}_{S S^{\prime}}}{v_{S S^{\prime}}^{2}} \vec{v}_{S S^{\prime}}-\frac{\sqrt{a^{2}-1}}{v_{S S^{\prime}}} c t_{S P} \vec{v}_{S S^{\prime}}, \\
t_{S^{\prime} P}=|a| t_{S P}-\frac{\sqrt{a^{2}-1}}{v_{S S^{\prime}}} \vec{x}_{S P} \cdot \vec{v}_{S S^{\prime}}, \\
\tau:=(1-|a|) \frac{\vec{x}_{S P} \cdot \vec{v}_{S S^{\prime}}}{v_{S S^{\prime}}^{2}}+\frac{\sqrt{a^{2}-1}}{v_{S S^{\prime}}} c t_{S P} .
\end{gathered}
$$

As we have expected, the transformation (23) satisfies the condition $\vec{x}_{S^{\prime} P}^{2}-c^{2} t_{S^{\prime} P}^{2}=\vec{x}_{S P}^{2}-c^{2} t_{S P}^{2}$ and leaves $\tau$ invariant, that is,

$$
\begin{aligned}
\tau^{\prime} & =(1-|a|) \frac{\vec{x}_{S^{\prime} P} \cdot \vec{v}_{S^{\prime} S}}{v_{S^{\prime} S}^{2}}+\frac{\sqrt{a^{2}-1}}{v_{S^{\prime} S}} c t_{S^{\prime} P} \\
& =(1-|a|) \frac{\vec{x}_{S P} \cdot \vec{v}_{S S^{\prime}}}{v_{S S^{\prime}}^{2}}+\frac{\sqrt{a^{2}-1}}{v_{S S^{\prime}}} c t_{S P}=\tau
\end{aligned}
$$

since it was built in order to satisfy those assumptions. Here, we assume that the parameter $a$ depends on the relative speed $v_{S S^{\prime}}$, which for convenience we denote by $a_{v_{S s^{\prime}}}$. Under this assumption, we also obtain that $a_{v_{S^{\prime} S}}=a_{v_{s S^{\prime}}}$.

The transformation (23) depends on the velocity $\vec{v}_{S S^{\prime}}$ that is defined as the derivative relative to the absolute time:

$$
\vec{v}_{S S^{\prime}}=\frac{d \vec{x}_{S S^{\prime}}}{d \tau}
$$

Now, considering the physical time $t_{S S^{\prime}}$ we can define another velocity

$$
\overrightarrow{\widetilde{v}}_{S S^{\prime}}:=\frac{d \vec{x}_{S S^{\prime}}}{d t_{S S^{\prime}}}
$$

We obtain a relation between $\overrightarrow{\widetilde{v}}_{S S^{\prime}}, \vec{v}_{S S^{\prime}}$ as follows. Identifying $P$ with the origin of the frame $S^{\prime}$ we have from (24)

$$
\tau=\frac{\sqrt{a_{v_{S s^{\prime}}}^{2}-1}}{\left|a_{v_{s s^{\prime}}}\right|} \frac{c}{v_{S S^{\prime}}} t_{S S^{\prime}}
$$

which allows us to write

$$
\vec{x}_{S S^{\prime}}=\vec{v}_{S S^{\prime}} \frac{\sqrt{a_{v_{S S^{\prime}}}^{2}-1}}{\left|a_{v_{S S^{\prime}}}\right|} \frac{c}{v_{S S^{\prime}}} t_{S S^{\prime}}
$$

and then

$$
\overrightarrow{\widetilde{v}}_{S S^{\prime}}=\vec{v}_{S S^{\prime}} \frac{\sqrt{a_{v_{S S^{\prime}}}^{2}-1}}{\left|a_{v_{S S^{\prime}}}\right|} \frac{c}{v_{S S^{\prime}}} .
$$

Since the parameter $a_{v_{S S^{\prime}}}$ depends on $v_{S S^{\prime}}$, we obtain that $\widetilde{v}_{S S^{\prime}}$ also depends on $v_{S S^{\prime}}$ through the expression

$$
\frac{\widetilde{v}_{S S^{\prime}}^{2}}{c^{2}}=\frac{a_{v_{S S^{\prime}}}^{2}-1}{a_{v_{S S^{\prime}}}^{2}}
$$


which gives

$$
\widetilde{v}_{S S^{\prime}}<c
$$

Therefore, in our formalism every speed calculated as the derivative relative to the physical time is always less than $c$. Since in SR this is the type of derivative we consider, it seems that the problem of tachyons is ruled out in SR. From (31) we may express $a_{v_{S s^{\prime}}}$ in terms of $\widetilde{v}_{S S^{\prime}}$ as

$$
a_{v_{S S^{\prime}}}=\frac{1}{\sqrt{1-\left(\widetilde{v}_{S S^{\prime}}^{2} / c^{2}\right)}} \equiv \gamma_{\widetilde{v}_{S S^{\prime}}},
$$

and the transformation (23) becomes

$$
\begin{gathered}
\vec{x}_{S^{\prime} P}=\vec{x}_{S P}-\left(1-\gamma_{\tilde{v}_{S S^{\prime}}}\right) \frac{\vec{x}_{S P} \cdot \overrightarrow{\widetilde{v}}_{S S^{\prime}}}{\widetilde{v}_{S S^{\prime}}^{2}} \overrightarrow{\vec{v}}_{S S^{\prime}}-\gamma_{\tilde{v}_{S S^{\prime}}} t_{S P} \overrightarrow{\vec{v}}_{S S^{\prime}}, \\
t_{S^{\prime} P}=\gamma_{\tilde{v}_{S S^{\prime}}}\left(t_{S P}-\frac{\vec{x}_{S P} \cdot \overrightarrow{\widetilde{v}}_{S S^{\prime}}}{c^{2}}\right),
\end{gathered}
$$

that is, the familiar Lorentz transformation. Hence, we have obtained a complete equivalence between the Galilei and the Lorentz transformation.

From (30) we obtain a similar expression relating $\overrightarrow{\widetilde{v}}_{S^{\prime} S}$ and $\vec{v}_{S^{\prime} S}$, that is, $\widetilde{v}_{S^{\prime} S}^{2} / c^{2}=\left(a_{v_{S^{\prime} S}}^{2}-1\right) / a_{v_{S^{\prime} S}}^{2}$, and since $a_{v_{S^{\prime} S}}$ depends on the absolute value $\left|\vec{v}_{S^{\prime} S}\right|=\left|\vec{v}_{S S^{\prime}}\right|$, we end up with

$$
\widetilde{v}_{S^{\prime} S}=\widetilde{v}_{S S^{\prime}} .
$$

From (30) we could also have considered $\overrightarrow{\widetilde{v}}_{S S^{\prime}}$ as a vector in $\mathbb{R}_{S S^{\prime}}^{3}$. However, we avoid this interpretation as it would lead to an inconsistency associated to the so-called Thomas precession that we will analyze in Section 3.4.3.

3.3. On the Role of the Parameter $a$. As we have seen, the parameter a was introduced in (18) as a free parameter in the sense that it was assumed to depend only on the relative speed $v_{S S^{\prime}}$ between the frames. The assumptions give no prescription on how to fix this dependence. Having established the relation between the absolute and the physical time, we introduced another velocity $\overrightarrow{\widetilde{v}}_{S S^{\prime}}$, considered as a derivative relative to the physical time, which is related to $\vec{v}_{S S^{\prime}}$ and the parameter $a_{v_{S s^{\prime}}}$ through (30), (31). Whatever the form we may take for $a_{v_{S S^{\prime}}}$, once we obtain $\widetilde{v}_{S S^{\prime}}$ as a function of $v_{S S^{\prime}}$ we may rewrite $a_{v_{S S^{\prime}}}$ in terms of $\widetilde{v}_{S S^{\prime}}$, which results in $\mathrm{a}_{v_{S S^{\prime}}}=$ $\gamma_{\tilde{v}_{s s^{\prime}}}(33)$. This gives a "universal" character for the Lorentz transformation with the transformation (23) becoming (34). Despite this, the constraint between $\vec{v}_{S S^{\prime}}$ and $\overrightarrow{\widetilde{v}}_{S S^{\prime}}$ arising from a particular choice of $a_{v_{S s^{\prime}}}$ may signalize different behaviors.

In fact, let us assume $v_{S S^{\prime}}>c$ and choose $a$ such that

$$
a_{v_{S S^{\prime}}} \equiv \tilde{\gamma}:=\frac{1}{\sqrt{1-\left(c^{2} / v_{S S^{\prime}}^{2}\right)}} .
$$

With this choice, the transformation (23) becomes

$$
\begin{gathered}
\vec{x}_{S^{\prime} P}=\vec{x}_{S P}-(1-\tilde{\gamma}) \frac{\vec{x}_{S P} \cdot \vec{v}_{S S^{\prime}}}{v_{S S^{\prime}}^{2}} \vec{v}_{S S^{\prime}}-\tilde{\gamma} \frac{c^{2}}{v_{S S^{\prime}}^{2}} t_{S P} \vec{v}_{S S^{\prime}}, \\
t_{S^{\prime} P}=\tilde{\gamma}\left(t_{S P}-\frac{\vec{x}_{S P} \cdot \vec{v}_{S S^{\prime}}}{v_{S S^{\prime}}^{2}}\right)
\end{gathered}
$$

that agrees with the expression obtained originally by Shankara (see $[9$, equations $(16),(17)]$ ) and also by Duffey [10] in the description of superluminal frames. From (31) we obtain

$$
\widetilde{v}_{S S^{\prime}}=\frac{c^{2}}{v_{S S^{\prime}}},
$$

a relation that is also present in the tachyonic model of [9], whose context is of a wave propagating in a medium with the velocities being interpreted in a different way; namely, $\overrightarrow{\widetilde{v}}_{S S^{\prime}}$ is the group velocity of the wave, while $\vec{v}_{S S^{\prime}}$ is the phase velocity. Here, even though the transformation (37) reduces to the form given on (34), which has the usual Lorentz form, the physical distinction between $\vec{v}_{S S^{\prime}}$ and $\overrightarrow{\widetilde{v}}_{S S^{\prime}}$ may be employed to select one of the forms of the transformation as being physically relevant for the problem one is analyzing. We return to this issue in Section 4.3.2.

3.4. The Velocity Transformation. Following the program of deducing transformations directly from the assumptions given in (I)-(IV) and the results previously obtained, we now intend to obtain the law of velocity transformation and analyze some of its consequences.

3.4.1. Obtaining the Transformation from the Galilean Velocity $L a w$. Let us assume two frames $S$ and $S^{\prime}$ moving with velocity $\vec{v}_{S S^{\prime}}$ and such that at $\tau=0$ (or equivalently at $t=0=t^{\prime}$ ) both origins coincide. The position of a moving particle is written as $\vec{x}_{S P}(\tau), \vec{x}_{S^{\prime} P}(\tau)$, and from $\vec{x}_{S^{\prime} P}=\vec{x}_{S P}-\vec{v}_{S S^{\prime}} \tau$ we obtain the rule of velocity addition in Galilei relativity:

$$
\frac{d \vec{x}_{S^{\prime} P}}{d \tau}=\frac{d \vec{x}_{S P}}{d \tau}-\vec{v}_{S S^{\prime}} .
$$

From (24), and using the chain rule, we have

$$
\begin{aligned}
& \left(\frac{d \vec{x}_{S^{\prime} P}}{d t_{S^{\prime} P}}\right)\left(-\left(1-\left|a_{v_{S S^{\prime}}}\right|\right) \frac{d \vec{x}_{S^{\prime} P}}{d t_{S^{\prime} P}} \cdot \frac{\vec{v}_{S S^{\prime}}}{v_{S S^{\prime}}^{2}}+\frac{\sqrt{a_{v_{S S^{\prime}}}^{2}-1}}{v_{S S^{\prime}}} c\right)^{-1} \\
& =\left(\frac{d \vec{x}_{S P}}{d t_{S P}}\right)\left(\left(1-\left|a_{v_{S S^{\prime}}}\right|\right) \frac{d \vec{x}_{S P}}{d t_{S P}} \cdot \frac{\vec{v}_{S S^{\prime}}}{v_{S S^{\prime}}^{2}}+\frac{\sqrt{a_{v_{S S^{\prime}}}^{2}-1}}{v_{S S^{\prime}}} c\right)^{-1} \\
& -\vec{v}_{S S^{\prime}} .
\end{aligned}
$$

Let us denote

$$
\overrightarrow{\widetilde{v}}_{S P} \equiv \frac{d \vec{x}_{S P}}{d t_{S P}}, \quad \overrightarrow{\widetilde{v}}_{S^{\prime} P} \equiv \frac{d \vec{x}_{S^{\prime} P}}{d t_{S^{\prime} P}} .
$$


Taking the scalar product by $\vec{v}_{S S^{\prime}}$ on both sides of (40) we obtain

$$
\begin{aligned}
& \overrightarrow{\widetilde{v}}_{S^{\prime} P} \cdot \vec{v}_{S S^{\prime}} \\
& =c v_{S S^{\prime}} \frac{\left|a_{v_{S S^{\prime}}}\right| \overrightarrow{\widetilde{v}}_{S P} \cdot \vec{v}_{S S^{\prime}}-\sqrt{a_{v_{S S^{\prime}}^{2}-1}} c v_{S S^{\prime}}}{-\sqrt{a_{v_{S S^{\prime}}}^{2}-1} \overrightarrow{\widetilde{v}}_{S P} \cdot \vec{v}_{S S^{\prime}}+\left|a_{v_{S S^{\prime}}}\right| c v_{S S^{\prime}}} .
\end{aligned}
$$

Using this last expression back in (40) together with (30) and (31), we obtain the final form

$$
\begin{aligned}
& \overrightarrow{\widetilde{v}}_{S^{\prime} P} \\
& =\frac{\overrightarrow{\widetilde{v}}_{S P}-\gamma_{\tilde{v}_{S S^{\prime}}} \overrightarrow{\widetilde{v}}_{S S^{\prime}}-\left(1-\gamma_{\tilde{v}_{S S^{\prime}}}\right)\left(\left(\overrightarrow{\widetilde{v}}_{S P} \cdot \overrightarrow{\widetilde{v}}_{S S^{\prime}}\right) / \widetilde{v}_{S S^{\prime}}^{2}\right) \overrightarrow{\widetilde{v}}_{S S^{\prime}}}{\gamma_{\widetilde{v}_{S S^{\prime}}}\left(1-\left(\left(\overrightarrow{\widetilde{v}}_{S P} \cdot \overrightarrow{\vec{v}}_{S S^{\prime}}\right) / c^{2}\right)\right)} .
\end{aligned}
$$

Since $\overrightarrow{\widetilde{v}}_{S^{\prime} P}$ and $\overrightarrow{\widetilde{v}}_{S P}$ are derivatives of the position vectors $\vec{x}_{S^{\prime} P}$, $\vec{x}_{S P}$ with respect to the physical times $t_{S^{\prime} P}$ and $t_{S P}$, we see (43) as the "physical time" counterpart of the rule of velocity addition of the Galilean relativity. It is straightforward to obtain that

$$
\frac{\widetilde{v}_{S^{\prime} P}^{2}}{c^{2}}=1+\frac{\left(\widetilde{v}_{S P}^{2} / c^{2}\right)-1}{\gamma_{\tilde{v}_{S S^{\prime}}}\left(1-\left(\left(\overrightarrow{\widetilde{v}}_{S P} \cdot \overrightarrow{\widetilde{v}}_{S S^{\prime}}\right) / c^{2}\right)\right)^{2}},
$$

and we conclude that $\tilde{v}_{S P}>c \Rightarrow \widetilde{v}_{S^{\prime} P}>c$ and $\widetilde{v}_{S P}<c \Rightarrow$ $\widetilde{v}_{S^{\prime} P}<c$. However, for a particle moving with a constant velocity relative to the frames $S, S^{\prime}$, we also have similar relations as the ones given in (31):

$$
\widetilde{v}_{S P}<c, \quad \widetilde{v}_{S^{\prime} P}<c .
$$

Therefore, the only possibility for having $\widetilde{v}_{S P}>c, \widetilde{v}_{S^{\prime} P}>c$ is related to a situation that does not demand relation (31), perhaps the case of an accelerated particle that would result in a relation for $\tau$ different from the one obtained in (24) that is the base for deducing (31).

3.4.2. The Light Speed as a Derivative Relative to $\tau$. Let us assume the propagation of a light ray with $\vec{x}_{S P}, \vec{x}_{S^{\prime} P}$ being the position of a point $P$ in the wave front as seen by $S$ and $S^{\prime}$; that is,

$$
\frac{d \vec{x}_{S P}}{d t_{S P}}=\vec{c}, \quad \frac{d \vec{x}_{S^{\prime} P}}{d t_{S^{\prime} P}}=\vec{c}^{\prime}
$$

with $\left|\vec{c}^{\prime}\right|=|\vec{c}|=c$. We then have

$$
\begin{aligned}
& \frac{d \vec{x}_{S P}}{d \tau}=\frac{d \vec{x}_{S P}}{d t_{S P}} \frac{d t_{S P}}{d \tau} \\
& \Longrightarrow c_{S}=\frac{c}{\left|(1-|a|)\left(\left(\vec{c} \cdot \vec{v}_{S S^{\prime}}\right) / v_{S S^{\prime}}^{2}\right)+\sqrt{a^{2}-1}\left(c / v_{S S^{\prime}}\right)\right|},
\end{aligned}
$$

$$
\begin{aligned}
& \frac{d \vec{x}_{S^{\prime} P}}{d \tau}=\frac{d \vec{x}_{S^{\prime} P}}{d t_{S^{\prime} P}} \frac{d t_{S^{\prime} P}}{d \tau} \\
& \Longrightarrow c_{S^{\prime}}=\frac{c}{\left|-(1-|a|)\left(\left(\vec{c}^{\prime} \cdot \vec{v}_{S S^{\prime}}\right) / v_{S S^{\prime}}^{2}\right)+\sqrt{a^{2}-1}\left(c / v_{S S^{\prime}}\right)\right|}
\end{aligned}
$$

that implies different values for the speed when considered as a rate of change relative to the absolute time; that is,

$$
c_{S}=\left|\frac{d \vec{x}_{S P}}{d \tau}\right| \neq c_{S}^{\prime}=\left|\frac{d \vec{x}_{S^{\prime} P}}{d \tau}\right| .
$$

Here, there is one more consistency check to be performed. We expect to have the following relation: $\left|\vec{x}_{S P}\right|=c_{S} \Delta \tau=c \Delta t$. In fact, consider an event $P:(t, \vec{x}) \sim(\tau, \vec{x})$ as described by $S$. At this instant, assume that the observer in the frame $S$ that is at the same location of the event $P$ sends a light ray towards the observer that is at the origin of $S$. The arrival of the light ray at the origin of $S$ corresponds to another event described by $S$ as $\left(t_{1}, 0\right) \sim\left(\tau_{1}, 0\right)$. Here, in order to relate $t$ and $\tau$, and $t_{1}$ and $\tau_{1}$, we assume any auxiliary frame $S^{\prime}$ moving relative to $S$ with velocity $\vec{v}$ and employ (25). For the events $(t, \vec{x}) \sim(\tau, \vec{x})$, $\left(t_{1}, 0\right) \sim\left(\tau_{1}, 0\right)$, we then have

$$
\begin{gathered}
\tau=(1-|a|) \frac{\vec{x} \cdot \vec{v}}{v^{2}}+\frac{\sqrt{a^{2}-1}}{v} c t, \\
\tau_{1}=\frac{\sqrt{a^{2}-1}}{v} c t_{1} .
\end{gathered}
$$

Denoting $\Delta t:=t_{1}-t$ and expressing $\vec{x}=-\vec{c} \Delta t$, we obtain

$$
\Delta \tau:=\tau_{1}-\tau=\left[\sqrt{a^{2}-1} \frac{c}{v}+(1-|a|) \frac{\vec{c} \cdot \vec{v}}{v^{2}}\right] \Delta t .
$$

Then,

$$
|\vec{x}|=c \Delta t=\frac{c}{\sqrt{a^{2}-1}(c / v)+(1-|a|)\left((\vec{c} \cdot \vec{v}) / v^{2}\right)} \Delta \tau,
$$

and from (47) we obtain $|\vec{x}|=c_{S} \Delta \tau$.

3.4.3. The Composition of Velocities. Let us assume three frames $\Sigma, S, S^{\prime}$ moving relative to each other with velocities $\vec{v}_{\Sigma S}, \vec{v}_{\Sigma S^{\prime}}, \vec{v}_{S S^{\prime}}$, having their axes parallel and their origins coinciding at $\tau=0$. According to Galilei relativity, we write

$$
\vec{x}_{\Sigma S^{\prime}}=\vec{x}_{\Sigma S}+\vec{x}_{S S^{\prime}}
$$

with

$$
\vec{x}_{\Sigma S^{\prime}}=\vec{v}_{\Sigma S^{\prime}} \tau, \quad \vec{x}_{\Sigma S}=\vec{v}_{\Sigma S} \tau, \quad \vec{x}_{S S^{\prime}}=\vec{v}_{S S^{\prime}} \tau .
$$

In particular, we have $\vec{x}_{\Sigma S^{\prime}}=-\vec{x}_{S^{\prime} \Sigma}$, which results in

$$
\vec{v}_{\Sigma S^{\prime}}=-\vec{v}_{S^{\prime} \Sigma}
$$

We now analyze the movement of $S^{\prime}$ relative to $S$ and $\Sigma$ and then the movement of $\Sigma$ relative to $S$ and $S^{\prime}$ using the same procedure that led to (43). 
(i) The Movement of $S^{\prime}$ Relative to $\Sigma$ and $S$. For the movement of $S^{\prime}$ relative to $\Sigma$ and $S$, we have $\left(t_{\Sigma S^{\prime}}, \vec{x}_{\Sigma S^{\prime}}\right) \sim\left(t_{S S^{\prime}}, \vec{x}_{S S^{\prime}}\right)$ and

$$
\tau=\left\{\begin{array}{l}
\left(1-\left|a_{v_{\Sigma S}}\right|\right) \frac{\vec{x}_{S S^{\prime}} \cdot\left(-\vec{v}_{\Sigma S}\right)}{v_{\Sigma S}^{2}}+\frac{\sqrt{a_{v_{\Sigma S}}^{2}-1}}{v_{\Sigma S}} c t_{S S^{\prime}} \\
\left(1-\left|a_{v_{\Sigma S}}\right|\right) \frac{\vec{x}_{\Sigma S^{\prime}} \cdot \vec{v}_{\Sigma S}}{v_{\Sigma S}^{2}}+\frac{\sqrt{a_{v_{\Sigma S}}^{2}-1}}{v_{\Sigma S}} c t_{\Sigma S^{\prime}} .
\end{array}\right.
$$

From (53) we obtain

$$
\frac{d \vec{x}_{\Sigma S^{\prime}}}{d t_{\Sigma S^{\prime}}} \frac{d t_{\Sigma S^{\prime}}}{d \tau}=\vec{v}_{\Sigma S}+\frac{d \vec{x}_{S S^{\prime}}}{d t_{S S^{\prime}}} \frac{d t_{S S^{\prime}}}{d \tau}
$$

Denoting

$$
\overrightarrow{\widetilde{v}}_{\Sigma S^{\prime}} \equiv \frac{d \vec{x}_{\Sigma S^{\prime}}}{d t_{\Sigma S^{\prime}}}, \quad \overrightarrow{\widetilde{v}}_{S S^{\prime}} \equiv \frac{d \vec{x}_{S S^{\prime}}}{d t_{S S^{\prime}}}
$$

and using (56), we obtain by the same procedure employed in obtaining (43) the following expression:

$$
\overrightarrow{\widetilde{v}}_{\Sigma S^{\prime}}=\frac{\overrightarrow{\widetilde{v}}_{S S^{\prime}}+\gamma_{\tilde{v}_{\Sigma S}} \overrightarrow{\widetilde{v}}_{\Sigma S}-\left(1-\gamma_{\widetilde{v}_{\Sigma S}}\right)\left(\left(\overrightarrow{\widetilde{v}}_{S S^{\prime}} \cdot \overrightarrow{\widetilde{v}}_{\Sigma S}\right) / \widetilde{v}_{\Sigma S}^{2}\right) \overrightarrow{\widetilde{v}}_{\Sigma S}}{\gamma_{\tilde{v}_{\Sigma S}}\left(1+\left(\left(\overrightarrow{\widetilde{v}}_{S S^{\prime}} \cdot \overrightarrow{\widetilde{v}}_{\Sigma S}\right) / c^{2}\right)\right)} .
$$

(ii) The Movement of $\Sigma$ Relative to $S$ and $S^{\prime}$. For the movement of $\Sigma$ relative to $S$ and $S^{\prime}$, we have $\left(t_{S \Sigma}, \vec{x}_{S \Sigma}\right) \sim\left(t_{S^{\prime} \Sigma}, \vec{x}_{S^{\prime} \Sigma}\right)$ and

$$
\tau=\left\{\begin{array}{l}
\left(1-\left|a_{v_{S^{\prime} S}}\right|\right) \frac{\vec{x}_{S \Sigma} \cdot\left(-\vec{v}_{S^{\prime} S}\right)}{v_{S^{\prime} S}^{2}}+\frac{\sqrt{a_{v_{S^{\prime} S}}^{2}-1}}{v_{S^{\prime} S}} c t_{S \Sigma}, \\
\left(1-\left|a_{v_{S^{\prime} S}}\right|\right) \frac{\vec{x}_{S^{\prime} \Sigma} \cdot \vec{v}_{S^{\prime} S}}{v_{S^{\prime} S}^{2}}+\frac{\sqrt{a_{s_{S \prime S}}^{2}-1}}{v_{S^{\prime} S}} c t_{S^{\prime} \Sigma} .
\end{array}\right.
$$

From (53) and using that $\vec{x}_{S^{\prime} \Sigma}=-\vec{x}_{\Sigma S^{\prime}}, \vec{x}_{\Sigma S}=-\vec{x}_{S \Sigma}, \vec{x}_{S^{\prime} S}=$ $-\vec{x}_{S S^{\prime}}$, we now have

$$
\frac{d \vec{x}_{S^{\prime} \Sigma}}{d t_{S^{\prime} \Sigma}} \frac{d t_{S^{\prime} \Sigma}}{d \tau}=\frac{d \vec{x}_{S \Sigma}}{d t_{S \Sigma}} \frac{d t_{S \Sigma}}{d \tau}+\vec{v}_{S^{\prime} S}
$$

Denoting

$$
\overrightarrow{\widetilde{v}}_{S^{\prime} \Sigma} \equiv \frac{d \vec{x}_{S^{\prime} \Sigma}}{d t_{S^{\prime} \Sigma}}, \quad \overrightarrow{\widetilde{v}}_{S \Sigma} \equiv \frac{d \vec{x}_{S \Sigma}}{d t_{S \Sigma}}
$$

we obtain

$$
\overrightarrow{\widetilde{v}}_{S^{\prime} \Sigma}=\frac{\overrightarrow{\widetilde{v}}_{S \Sigma}+\gamma_{\tilde{v}_{S S^{\prime}}} \overrightarrow{\vec{v}}_{S^{\prime} S}-\left(1-\gamma_{\widetilde{v}_{S S^{\prime}}}\right)\left(\left(\overrightarrow{\widetilde{v}}_{S \Sigma} \cdot \overrightarrow{\widetilde{v}}_{S^{\prime} S}\right) / \widetilde{v}_{S^{\prime} S}^{2}\right) \overrightarrow{\widetilde{v}}_{S^{\prime} S}}{\gamma_{\tilde{v}_{S S^{\prime}}}\left(1+\left(\left(\overrightarrow{\widetilde{v}}_{S \Sigma} \cdot \overrightarrow{\widetilde{v}}_{S^{\prime} S}\right) / c^{2}\right)\right)},
$$

where we have used that $\widetilde{v}_{S S^{\prime}}=\widetilde{v}_{S^{\prime} S}$ to identify $\gamma_{\tilde{v}_{S S^{\prime}}}=\gamma_{\widetilde{v}_{S^{\prime} S}}$. We recognize (59), (63) with the velocity transformations obtained by Møller in his analysis of the Thomas precession [5]. As we know, in this particular case, the velocities $\overrightarrow{\widetilde{v}}_{\Sigma S^{\prime}}, \overrightarrow{\widetilde{v}}_{S^{\prime} \Sigma}$ are related by a rotation $[5,11]$. We notice that each of $(59)$,
(63) involves vectors belonging to different spaces; therefore we should interpret each equation in the same way as we did for (9); that is, $\overrightarrow{\widetilde{v}}_{\Sigma S^{\prime}}, \overrightarrow{\widetilde{v}}_{S^{\prime} \Sigma}$ are vectors belonging to an abstract space $V$ where (59), (63) make sense. It is in this space that we have

$$
\overrightarrow{\widetilde{v}}_{\Sigma S^{\prime}} \neq-\overrightarrow{\widetilde{v}}_{S^{\prime} \Sigma}
$$

which accounts for the Thomas precession. However, the vectors $\vec{v}_{\Sigma S^{\prime}}, \vec{v}_{S^{\prime} \Sigma}$ are defined in the space $\mathbb{R}_{\Sigma S S^{\prime}}^{3}$ where they satisfy (55), which is a consequence of the frames $\Sigma, S, S^{\prime}$ having their axis parallel during its translational movement. Now, the reason for not identifying the velocities $\overrightarrow{\widetilde{v}}_{\Sigma S^{\prime}}, \overrightarrow{\widetilde{v}}_{S^{\prime} \Sigma}$ as vectors in $\mathbb{R}_{\Sigma S S^{\prime}}^{3}$ becomes clear. Indeed, if this was the case, then using (30) we would associate each of them with the vectors $\vec{v}_{\Sigma S^{\prime}}, \vec{v}_{S^{\prime} \Sigma}$ through

$$
\overrightarrow{\widetilde{v}}_{\Sigma S^{\prime}}=\vec{v}_{\Sigma S^{\prime}} \frac{\sqrt{a_{v_{\Sigma S^{\prime}}}^{2}-1}}{\left|a_{v_{\Sigma S^{\prime}}}\right|} \frac{c}{v_{\Sigma S^{\prime}}}, \quad \overrightarrow{\widetilde{v}}_{S^{\prime} \Sigma}=\vec{v}_{S^{\prime} \Sigma} \frac{\sqrt{a_{v_{S^{\prime} \Sigma}}^{2}-1}}{\left|a_{v_{S^{\prime} \Sigma}}\right|} \frac{c}{v_{S^{\prime} \Sigma}},
$$

and from (55) we would have $\overrightarrow{\widetilde{v}}_{\Sigma S^{\prime}}=-\overrightarrow{\widetilde{v}}_{S^{\prime} \Sigma}$, which contradicts (64).

3.5. The Structure of Space and Time. Spacetime is the physical arena where events occur. Therefore, in order to describe spacetime, we must endow it with a suitable coordinate system, for example, the one provided by a reference frame, which is how we describe events. In our approach, we have an equivalence between the absolute and the physical time that allows an observer to associate coordinates with events according to $(\tau, \vec{x})$ or $(t, \vec{x})$, and from each of these coordinatizations originates a particular view for the spacetime. In what follows, we search for a description of spacetime that combines the views of the Galilei and the Special relativities.

Given two frames $S, S^{\prime}$, let us write the Galilei transformation as

$$
\left(\tau_{S}, \vec{x}_{S}\right) \longrightarrow\left(\tau_{S^{\prime}}, \vec{x}_{S^{\prime}}\right):\left\{\begin{array}{l}
\tau_{S}=\tau_{S^{\prime}} \equiv \tau \\
\vec{x}_{S^{\prime}}=\vec{x}_{S}-\vec{v} \tau
\end{array}\right.
$$

Due to the absolute character of $\tau$, we have that for a fixed $\tau \equiv$ $\tau_{S}=\tau_{S^{\prime}}$ the Galilei transformation is essentially a map $\mathbb{R}_{S}^{3} \rightarrow$ $\mathbb{R}_{S^{\prime}}^{3}$, where $\mathbb{R}_{S}^{3} \ni \vec{x}_{S}, \mathbb{R}_{S^{\prime}}^{3} \ni \vec{x}_{S^{\prime}}$ denote the 3-dimensional Euclidean space. When spacetime is described using $(\tau, \vec{x})$ as coordinates, we refer to it as the Galilei spacetime that admits the following representation: $\cup_{\tau \in \mathbb{R}}\left(\{\tau\} \times \mathbb{R}^{3}\right) \equiv \mathbb{R} \times \mathbb{R}^{3}$. In this sense, the Galilei spacetime decomposes in terms of 3dimensional spaces $\mathbb{R}^{3}$ that are indexed by $\tau \in \mathbb{R}$.

Now, let us analyze the transformation under the form (23). For the same fixed value of $\tau$, we rewrite (25) as

$$
\tau=x^{0} n^{0}+\vec{x} \cdot \vec{n}=x^{\prime 0} n^{\prime 0}+\vec{x}^{\prime} \cdot \vec{n}^{\prime}
$$

with

$$
\begin{aligned}
& n^{\mu} \equiv\left(n^{0}, \vec{n}\right):=\left(\frac{\sqrt{a^{2}-1}}{v},-\frac{(|a|-1) \vec{v}}{v^{2}}\right) \\
& n^{\prime \mu} \equiv\left(n^{\prime 0}, \vec{n}^{\prime}\right):=\left(\frac{\sqrt{a^{2}-1}}{v}, \frac{(|a|-1) \vec{v}}{v^{2}}\right)
\end{aligned}
$$


and $x^{\mu} \equiv\left(x^{0}, \vec{x}\right):=(c t, \vec{x}), x^{\prime \mu} \equiv\left(x^{\prime 0}, \vec{x}^{\prime}\right):=\left(c t^{\prime}, \vec{x}^{\prime}\right)$. We identify (67) as the equation of two hyperplanes $\sigma_{\tau}$ : $\tau=x^{0} n^{0}+\vec{x} \cdot \vec{n}$ and $\sigma_{\tau}^{\prime}: \tau=x^{\prime 0} n^{\prime 0}+\vec{x}^{\prime} \cdot \vec{n}^{\prime}$ defined, respectively, in the spaces $\mathbb{R}_{S}^{4}$ and $\mathbb{R}_{S^{\prime}}^{4}$ (the spaces $\mathbb{R}_{S}^{4} \ni(c t, \vec{x})$, $\mathbb{R}_{S^{\prime}}^{4} \ni\left(c t^{\prime}, \vec{x}^{\prime}\right)$ serve here as the background in terms of which each observer describes spacetime). Then, for a fixed value of $\tau$, the transformation (23) is seen as a transformation $\sigma_{\tau} \rightarrow \sigma_{\tau}^{\prime}$ between these hyperplanes. Here, when spacetime is described using $(t, \vec{x})$ as coordinates (in fact, in order to have coordinates with the dimension of length we would write $(c t, \vec{x})$ and $(c \tau, \vec{x})$ in place of, respectively, $(t, \vec{x})$ and $(\tau, \vec{x}))$, we refer to it as the SR spacetime and it decomposes as $\cup_{\tau \in \mathbb{R}} \sigma_{\tau} \equiv \mathbb{R}^{4}$.

From the previous considerations, the properties of the spacetime that arise from our unified model are summarized as follows. First, we notice that the Galilei and SR spacetime refer to the same spacetime in fact, they are just different descriptions of the same structure. Second, both the Galilei and the SR spacetime are sliced by 3-dimensional spaces, with the Galilei spacetime being "sliced" by hyperplanes that are normal to the $\tau$-direction or, equivalently, by planes that correspond to the space $\mathbb{R}^{3}$. For the SR spacetime, the "slicing" is made by hyperplanes $\sigma_{\tau}, \sigma_{\tau}^{\prime}$ that are orthogonal to the direction of the vectors $n$ and $n^{\prime}$. In particular, the relation between the Galilei transformation and the Lorentz transformation is clarified by the diagram below:

$$
\begin{array}{cc}
\mathbb{R}_{S}^{3} \ni(\tau, \vec{x}) \stackrel{i_{\tau}}{\longrightarrow}(c t(\tau, \vec{x}), \vec{x}) \in \sigma_{\tau} \subset \mathbb{R}_{S}^{4} \\
\downarrow \varphi_{G} & \downarrow \varphi_{L} \\
\mathbb{R}_{S^{\prime}}^{3} \ni\left(\tau, \vec{x}^{\prime}\right) \stackrel{i_{\tau}^{\prime}}{\longrightarrow}\left(c t^{\prime}\left(\tau, \vec{x}^{\prime}\right), \vec{x}^{\prime}\right) \in \sigma_{\tau}^{\prime} \subset \mathbb{R}_{S^{\prime}}^{4}
\end{array}
$$

where for a fixed $\tau$ and relative to two frames of reference $S, S^{\prime}$ that move with relative velocity $\vec{v}$ we have defined mappings

$$
\begin{gathered}
i_{\tau}: \mathbb{R}_{S}^{3} \longrightarrow \mathbb{R}_{S^{4},}^{4} \\
\vec{x} \longrightarrow(c t(\tau, \vec{x}), \vec{x}), \\
t(\tau, \vec{x})=\frac{v}{c \sqrt{a^{2}-1}}\left(\tau+(|a|-1) \frac{\vec{x} \cdot \vec{v}}{v^{2}}\right), \\
i_{\tau}^{\prime}: \mathbb{R}_{S^{\prime}}^{3} \longrightarrow \mathbb{R}_{S^{\prime}}^{4}, \\
\vec{x}^{\prime} \longrightarrow\left(c t^{\prime}\left(\tau, \vec{x}^{\prime}\right), \vec{x}^{\prime}\right), \\
t^{\prime}\left(\tau, \vec{x}^{\prime}\right)=\frac{v}{c \sqrt{a^{2}-1}}\left(\tau-(|a|-1) \frac{\vec{x}^{\prime} \cdot \vec{v}}{v^{2}}\right) .
\end{gathered}
$$

We notice that endowing $\mathbb{R}_{S}^{3}, \mathbb{R}_{S^{\prime}}^{3}, \mathbb{R}_{S}^{4}, \mathbb{R}_{S^{\prime}}^{4}$ with the standard Euclidean topology we obtain $i_{\tau}\left(\mathbb{R}_{S}^{3}\right)=\sigma_{\tau}, i_{\tau}^{\prime}\left(\mathbb{R}_{S^{\prime}}^{3}\right)=\sigma_{\tau}^{\prime}$ as embedded, respectively, in $\mathbb{R}_{S}^{4}$ and $\mathbb{R}_{\mathcal{S}^{\prime}}^{4}$. Then, given a Galilei transformation $(\tau, \vec{x}) \stackrel{\varphi_{G}}{\longrightarrow}\left(\tau, \vec{x}^{\prime}\right)$, the embeddings $i_{\tau}, i_{\tau}^{\prime}$ transfer $\varphi_{G}$ to the spaces $\mathbb{R}_{S}^{4}, \mathbb{R}_{S^{\prime}}^{4}$ where it assumes the form of the Lorentz transformation $(c t, \vec{x}) \stackrel{\varphi_{L}}{\longrightarrow}\left(c t^{\prime}, \vec{x}^{\prime}\right)$, which is shown in the scheme below

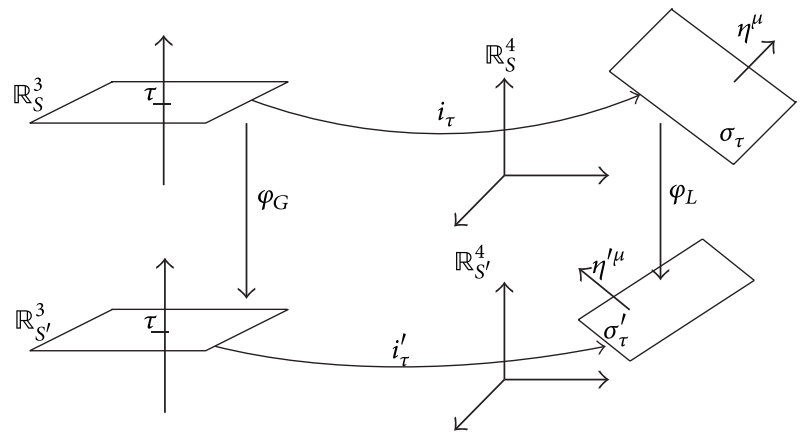

This view of the transformation provides an alternative way to analyze some aspects of the composition of Lorentz transformations. Indeed, let us write the Lorentz transformation as

$$
\varphi_{L}\left(v_{S S^{\prime}}\right)=i_{\tau}^{\prime}\left(v_{S S^{\prime}}\right) \circ \varphi_{G}\left(v_{S S^{\prime}}\right) \circ i_{\tau}^{-1}\left(v_{S S^{\prime}}\right)
$$

and consider three frames $S, S^{\prime}$, and $S^{\prime \prime}$ that move with relative velocities $\vec{v}_{S S^{\prime}}, \vec{v}_{S S^{\prime \prime}}$, and $\vec{v}_{S^{\prime} S^{\prime \prime}}$. Consider then the following diagram

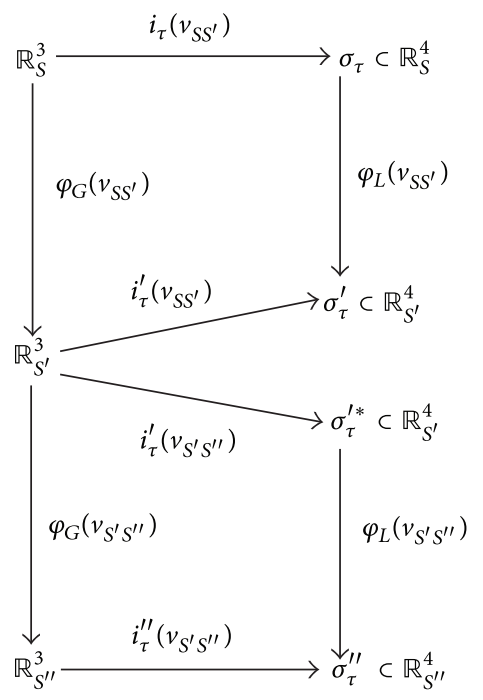

We notice that since the hyperplanes $\sigma_{\tau}^{\prime}$ and $\sigma_{\tau}^{\prime *}$ are different, the Lorentz transformations $\varphi_{L}\left(v_{S S^{\prime}}\right)$ and $\varphi_{L}\left(v_{S^{\prime} S^{\prime \prime}}\right)$ may be composed only through the maps $i_{\tau}^{\prime}\left(v_{S^{\prime} S^{\prime \prime}}\right) \circ i_{\tau}^{\prime-1}\left(v_{S S^{\prime}}\right)$, for example,

$$
\varphi_{L}\left(v_{S^{\prime} S^{\prime \prime}}\right) \circ i_{\tau}^{\prime}\left(v_{S^{\prime} S^{\prime \prime}}\right) \circ i_{\tau}^{\prime-1}\left(v_{S S^{\prime}}\right) \circ \varphi_{L}\left(v_{S S^{\prime}}\right)
$$

and we have

$$
\begin{aligned}
& \varphi_{L}\left(v_{S^{\prime} S^{\prime \prime}}\right) \circ i_{\tau}^{\prime}\left(v_{S^{\prime} S^{\prime \prime}}\right) \circ i_{\tau}^{-1}\left(v_{S S^{\prime}}\right) \circ \varphi_{L}\left(v_{S S^{\prime}}\right) \\
& \quad=i_{\tau}^{\prime \prime}\left(v_{S^{\prime} S^{\prime \prime}}\right) \circ \varphi_{G}\left(v_{S^{\prime} S^{\prime \prime}}\right) \circ \varphi_{G}\left(v_{S S^{\prime}}\right) \circ i_{\tau}^{-1}\left(v_{S S^{\prime}}\right) \\
& \quad=i_{\tau}^{\prime \prime}\left(v_{S^{\prime} S^{\prime \prime}}\right) \circ \varphi_{G}\left(v_{S S^{\prime \prime}}\right) \circ i_{\tau}^{-1}\left(v_{S S^{\prime}}\right) \\
& \quad \neq \varphi_{L}\left(v_{S S^{\prime \prime}}\right) .
\end{aligned}
$$


Therefore, from this interpretation of the Lorentz transformation acting on hyperplanes associated to the same value of $\tau$, we reobtain the common fact that the composition of two Lorentz transformations is not a Lorentz transformation. Here, the reason is that even though the composition $\varphi_{G}\left(v_{S^{\prime} S^{\prime \prime}}\right) \circ \varphi_{G}\left(v_{S S^{\prime}}\right)$ may be identified with $\varphi_{G}\left(v_{S S^{\prime \prime}}\right)$, the latter induces the Lorentz transformation $\varphi_{L}\left(v_{S S^{\prime \prime}}\right)$ only through the embeddings $i_{\tau}^{\prime \prime}\left(v_{S S^{\prime \prime}}\right), i_{\tau}^{-1}\left(v_{S S^{\prime \prime}}\right)$.

\section{Model II}

4.1. Assumptions. We propose another model based on the following assumptions.

(I) Space. Each inertial frame describes space as an euclidean 3-dimensional vector space.

(II) Time. Time is described by a variable $t$ that depends on the frame in such a way that two inertial frames $S$ and $S^{\prime}$ measure time $t$ and $t^{\prime}$.

(III) The speed of light is constant relative to inertial frames.

(IV) Relative to two inertial frames, any event $(t, \vec{x}) \sim$ $\left(t^{\prime}, \vec{x}^{\prime}\right)$ satisfies

$$
\alpha x^{0}+\vec{\beta} \cdot \vec{x}=\alpha x^{\prime 0}-\vec{\beta} \cdot \vec{x}^{\prime}
$$

with $\alpha, \vec{\beta}$ arbitrary parameters.

(V) The relation between $(t, \vec{x})$ and $\left(t^{\prime}, \vec{x}^{\prime}\right)$ is linear.

Remarks. (i) Postulates (I) and (II) are essentially the same postulates (I) and (II) of model I, except that in the current postulate (II) of model II there is no mention of the absolute time.

(ii) The condition $\alpha x^{0}+\vec{\beta} \cdot \vec{x}=\alpha x^{\prime 0}-\vec{\beta} \cdot \vec{x}^{\prime}$ is suggested by a previous condition given in (67), but now with the constants $\alpha, \vec{\beta}$ being arbitrary parameters. Here, (76) is the starting point from which we will derive the transformation. In this sense, it plays the same role as the relation $\vec{x}^{\prime 2}-c^{2} t^{\prime 2}=$ $\vec{x}^{2}-c^{2} t^{2}$ of the standard SR formulation. However, while in SR this last equation may be expressed in terms of the invariance of the quadratic form $Q\left(x^{\mu}\right):=\vec{x}^{2}-c^{2} t^{2}$, which is a quantity defined uniquely in terms of the spacetime coordinates, the same cannot be said of (76) since it contains explicitly the parameters $\alpha, \vec{\beta}$ that, in general, do not refer to any known property of the space and time. Therefore, unless we are able to identify the parameters $\alpha, \vec{\beta}$ with any intrinsic property of the spacetime, it will not be possible to associate any mathematical structure with (76) as it was done with $Q\left(x^{\mu}\right)$.

4.2. The Generalized Lorentz Transformation. From (V) we write

$$
\begin{aligned}
& x^{\prime 0}=A_{0}^{0} x^{0}+A_{i}^{0} x^{i}, \\
& x^{\prime i}=A_{0}^{i} x^{0}+A_{j}^{i} x^{j} .
\end{aligned}
$$

From (IV), we obtain by replacing them into (76) that

$$
\begin{gathered}
A_{0}^{0}=1+\frac{1}{\alpha} A_{0}^{i} \beta^{i}, \\
A_{i}^{0}=\frac{1}{\alpha}\left(\beta^{i}+A_{i}^{j} \beta^{j}\right)
\end{gathered}
$$

which momentarily gives

$$
\begin{gathered}
t^{\prime}=\left(1+\frac{1}{\alpha} A_{0}^{i} \beta^{i}\right) t+\frac{1}{\alpha c}\left(\beta^{i}+A_{i}^{j} \beta^{j}\right) x^{i}, \\
x^{i}=A_{0}^{i} c t+A_{j}^{i} x^{j} .
\end{gathered}
$$

Here we assume

$$
\begin{gathered}
A_{0}^{i}=\lambda \beta^{i}, \\
A_{j}^{i}=\delta_{j}^{i}+\xi \beta^{i} \beta^{j}
\end{gathered}
$$

with $\lambda, \xi$ arbitrary quantities that will be fixed later. We require that the inverse transformation is obtained by the change of $\left(t, \vec{x}, \beta^{i}\right) \leftrightarrow\left(t^{\prime}, \vec{x}^{\prime},-\beta^{i}\right)$ into (79). This fixes $\lambda=\xi \alpha$. Let us consider now the expression for the velocity. Here, we have

$$
\frac{d x^{\prime i}}{d t^{\prime}}=\frac{A_{0}^{i} c+A_{j}^{i}\left(d x^{j} / d t\right)}{1+(1 / \alpha) A_{0}^{i} \beta^{i}+(1 / \alpha c)\left(\beta^{i}+A_{i}^{j} \beta^{j}\right)\left(d x^{i} / d t\right)}
$$

From (III) the speed of light is constant relative to any inertial frame. Then for a light wave we have

$$
\frac{d \vec{x}^{\prime}}{d t^{\prime}}=\vec{c}^{\prime}, \quad \frac{d \vec{x}}{d t}=\vec{c} \equiv c \hat{\eta}
$$

with $\hat{\eta} \cdot \hat{\eta}=1, \quad\left|\vec{c}^{\prime}\right|=|\vec{c}|$.

From (81), (80), using $\lambda=\xi \alpha$, and due to the arbitrariness of the direction of the unit vector $\hat{\eta}$, we obtain

$$
\xi=\frac{2}{\alpha^{2}\left(1-\left(\beta^{2} / \alpha^{2}\right)\right)}
$$

which then fixes all the transformation constants $A_{0}^{0}, A_{i}^{0}, A_{0}^{i}$, and $A_{j}^{i}$ in terms of $\alpha, \beta$ as follows

$$
\begin{gathered}
A_{0}^{0}=\frac{1+\left(\beta^{2} / \alpha^{2}\right)}{1-\left(\beta^{2} / \alpha^{2}\right)}, \quad A_{i}^{0}=A_{0}^{i}=\frac{2}{1-\left(\beta^{2} / \alpha^{2}\right)} \frac{\beta^{i}}{\alpha}, \\
A_{j}^{i}=\delta_{j}^{i}+\frac{2}{1-\left(\beta^{2} / \alpha^{2}\right)} \frac{\beta^{i}}{\alpha} \frac{\beta^{j}}{\alpha}
\end{gathered}
$$

and finally leaves us with the transformations

$$
\begin{gathered}
t^{\prime}=\left(\frac{1+\left(\beta^{2} / \alpha^{2}\right)}{1-\left(\beta^{2} / \alpha^{2}\right)}\right)\left\{t+\frac{2}{c\left(1+\left(\beta^{2} / \alpha^{2}\right)\right)} \frac{\vec{\beta}}{\alpha} \cdot \vec{x}\right\}, \\
\vec{x}^{\prime}=\vec{x}+\frac{2}{1-\left(\beta^{2} / \alpha^{2}\right)} \frac{\vec{\beta}}{\alpha} \cdot \vec{x} \frac{\vec{\beta}}{\alpha}+\frac{2 c t}{1-\left(\beta^{2} / \alpha^{2}\right)} \frac{\vec{\beta}}{\alpha} .
\end{gathered}
$$


We have then obtained a class of transformations that is compatible with the invariance of the speed of light and that depends on arbitrary parameters $\alpha, \vec{\beta}$. We call this transformation Generalized Lorentz Transformation (GLT). It is immediate to check that it satisfies $\vec{x}^{\prime 2}-c^{2} t^{\prime 2}=\vec{x}^{2}-c^{2} t^{2}$ (and not only for a light wave), which shows that it must include the standard Lorentz transformation as a particular case.

Remark 1. It is possible to work with a more general relation than the one given in (76); for example, if we assume that $\vec{\beta}$ can be transformed, then we would write

$$
\alpha x^{0}+\vec{\beta} \cdot \vec{x}=\alpha x^{\prime 0}-\vec{\beta}^{\prime} \cdot \vec{x}^{\prime}
$$

and the effect of the invariance of $\vec{\beta}^{\prime} \cdot \vec{x}^{\prime}$ in this new condition corresponds to replace $\vec{\beta} \rightarrow \mathscr{R} \vec{\beta}, \vec{x} \rightarrow \mathscr{R} \vec{x}$ in (85), that is,

$$
\begin{aligned}
t^{\prime} & =\left(\frac{1+\left(\beta^{2} / \alpha^{2}\right)}{1-\left(\beta^{2} / \alpha^{2}\right)}\right)\left\{t+\frac{2}{c\left(1+\left(\beta^{2} / \alpha^{2}\right)\right)} \frac{\vec{\beta}}{\alpha} \cdot \vec{x}\right\}, \\
\vec{x}^{\prime} & =\mathscr{R} \vec{x}+\frac{2}{1-\left(\beta^{2} / \alpha^{2}\right)} \frac{\vec{\beta}}{\alpha} \cdot \vec{x} \frac{\mathscr{R} \vec{\beta}}{\alpha}+\frac{2 c t}{1-\left(\beta^{2} / \alpha^{2}\right)} \frac{\mathscr{R} \vec{\beta}}{\alpha}
\end{aligned}
$$

with $\mathscr{R}$ being an arbitrary space rotation.

In [12] Kapuscik using only the assumption of the linearity of the transformation and the constancy of the speed of light proposed a generalization of the Lorentz transformation having the form

$$
\begin{gathered}
t^{\prime}=A t+\vec{B} \cdot \vec{x} \\
\vec{x}^{\prime}=\sqrt{A^{2}-c^{2} \vec{B}^{2}}(\mathscr{R} \vec{x})+\frac{A-\sqrt{A^{2}-c^{2} \vec{B}^{2}}}{\vec{B}^{2}}(\mathscr{R} \vec{B})(\vec{B} \cdot \vec{x}) \\
+c^{2}(\mathscr{R} \vec{B}) t .
\end{gathered}
$$

The comparison of the time transformation given in (87) and (88) suggests the identification

$$
A=\frac{1+\left(\beta^{2} / \alpha^{2}\right)}{1-\left(\beta^{2} / \alpha^{2}\right)}, \quad \vec{B}=\frac{2}{c\left(1-\left(\beta^{2} / \alpha^{2}\right)\right)} \frac{\vec{\beta}}{\alpha}
$$

that also shows the equivalence of the space transformation. In our formalism, up to the derivation of (87), there is no restriction on the parameters $\alpha, \vec{\beta}$, while in the development of [12] they have to obey $A^{2}-c^{2} \vec{B}^{2}>0$. Since in [12] the relative velocity $\vec{V}$ between the frames is identified with $-c^{2}(\vec{B} / A)$, the previous inequality leads to the restriction $V<$ c. As we will show in Sections 4.3.1 and 4.3.2, in our formalism there is no restriction on the relative speed of the frames. Having obtained the GLT given in (85) we analyze in the sequence some of its properties.
4.3. Transformation of Velocity. Associated with GLR, we obtain by replacing (84) into (81) the associated transformation of velocity as

$$
\begin{aligned}
& \frac{d \vec{x}^{\prime}}{d t^{\prime}}=\left(\frac{d \vec{x}}{d t}+\frac{2 c}{1-\left(\beta^{2} / \alpha^{2}\right)} \frac{\vec{\beta}}{\alpha}+\frac{2}{1-\left(\beta^{2} / \alpha^{2}\right)} \frac{\vec{\beta}}{\alpha} \cdot \frac{d \vec{x}}{d t} \frac{\vec{\beta}}{\alpha}\right) \\
& \times\left(\frac{1+\left(\beta^{2} / \alpha^{2}\right)}{1-\left(\beta^{2} / \alpha^{2}\right)}+\frac{2}{c\left(1-\left(\beta^{2} / \alpha^{2}\right)\right)} \frac{\vec{\beta}}{\alpha} \cdot \frac{d \vec{x}}{d t}\right)^{-1} \\
&\left(\frac{d \vec{x}^{\prime}}{d t^{\prime}}\right)^{2}-c^{2} \\
&=\left.\left(\frac{d \vec{x}}{d t}\right)^{2}-c^{2}\right) \\
& \times\left(\left(\frac{1+\left(\beta^{2} / \alpha^{2}\right)}{1-\left(\beta^{2} / \alpha^{2}\right)}\right.\right. \\
&\left.\left.+\frac{2}{c\left(1-\left(\beta^{2} / \alpha^{2}\right)\right)} \frac{\vec{\beta}}{\alpha} \cdot \frac{d \vec{x}}{d t}\right)^{2}\right)^{-1}
\end{aligned}
$$

Therefore we obtain for the GLT that

$$
\left|\frac{d \vec{x}}{d t}\right|<c \Longleftrightarrow\left|\frac{d \vec{x}^{\prime}}{d t^{\prime}}\right|<c \quad \text { or } \quad\left|\frac{d \vec{x}}{d t}\right|>c \Longleftrightarrow\left|\frac{d \vec{x}^{\prime}}{d t^{\prime}}\right|>c .
$$

That is, relative to the two frames described by transformation (85), a particle that is described as subluminal (superluminal) particle by one frame will also be described as subluminal (superluminal) particle by the other frame.

4.3.1. A Transformation Involving Subluminal Frames: $\widetilde{v}<c$. As a particular case of transformation (85) let us choose the parameters $\alpha, \vec{\beta}$ in such way that

$$
\frac{1+\left(\beta^{2} / \alpha^{2}\right)}{1-\left(\beta^{2} / \alpha^{2}\right)}=\frac{1}{\sqrt{1-\left(\widetilde{v}^{2} / c^{2}\right)}}
$$

with $\widetilde{v}<c$. We obtain

$$
\frac{\vec{\beta}}{\alpha}=-c\left(1-\sqrt{1-\frac{\widetilde{v}^{2}}{c^{2}}}\right) \frac{\overrightarrow{\vec{v}}}{\widetilde{v}^{2}}
$$

that replacing in (85) leads to the transformation

$$
\begin{gathered}
t^{\prime}=\gamma\left(t-\frac{\vec{x} \cdot \overrightarrow{\vec{v}}}{c^{2}}\right), \\
\vec{x}^{\prime}=\vec{x}-(1-\gamma) \frac{\vec{x} \cdot \overrightarrow{\widetilde{v}}}{\widetilde{v}^{2}} \overrightarrow{\widetilde{v}}-\gamma t \overrightarrow{\widetilde{v}}
\end{gathered}
$$

with $\gamma:=1 / \sqrt{1-\left(\widetilde{v}^{2} / c^{2}\right)}$ that corresponds to the Lorentz transformation. 
4.3.2. A Transformation Involving Superluminal Frames: $\widetilde{v}>c$. Let us choose $\alpha, \vec{\beta}$ satisfying

$$
\frac{1+\left(\beta^{2} / \alpha^{2}\right)}{1-\left(\beta^{2} / \alpha^{2}\right)}=\frac{1}{\sqrt{1-\left(c^{2} / \widetilde{v}^{2}\right)}}
$$

with $\widetilde{v}>c$. Now we obtain

$$
\frac{\vec{\beta}}{\alpha}=-\frac{1}{c}\left(1-\sqrt{1-\frac{c^{2}}{\widetilde{v}^{2}}}\right) \overrightarrow{\widetilde{v}}
$$

and replacing it into (85) gives the transformation

$$
\begin{gathered}
t^{\prime}=\widetilde{\gamma}\left(t-\frac{\vec{x} \cdot \overrightarrow{\vec{v}}}{\widetilde{v}^{2}}\right), \\
\vec{x}^{\prime}=\vec{x}-(1-\widetilde{\gamma}) \frac{\vec{x} \cdot \overrightarrow{\widetilde{v}}}{\widetilde{\widetilde{v}}^{2}}-\widetilde{\gamma} c_{\widetilde{v}^{2}}^{2} t \overrightarrow{\widetilde{v}}
\end{gathered}
$$

with $\tilde{\gamma}:=1 / \sqrt{1-\left(c^{2} / \widetilde{v}^{2}\right)}$. Here, we reobtained Shankara and Duffey's transformation for tachyons $[9,10]$. This transformation (98) appeared originally in [9] in the description of the propagation of a wave in a medium with one of the frames being at rest relative to the medium and the other moving with a speed greater than $c$ (in fact, in [9] since the medium is not the vacuum, instead of $c$ the light speed is denoted by $w_{0}$ ). The physical content of this transformation has been discussed more generally in [10], to which we refer the reader for details. Here we wish to analyze the role played by the velocity $\overrightarrow{\widetilde{v}}$ in the transformation. We consider two situations.

First, let us consider the movement of an object such that according to the frame $S$ the object is at rest relative to the frame $S^{\prime}$. Assume that $\overrightarrow{\tilde{v}}$ is the relative velocity between the frames. Then the frame $S$ writes for the position of the object $\vec{x}=\overrightarrow{\tilde{v}} t$. Replacing it in (98), we obtain $t^{\prime}=0$ and $\vec{x}^{\prime}=(1 / \widetilde{\gamma}) t \overrightarrow{\vec{v}}=(1 / \widetilde{\gamma}) \vec{x}$. Therefore, for an interval $(0, \Delta t), S$ associates with the movement of the object the set of events $\{(t, \vec{x}):=(t, \overrightarrow{\widetilde{v}} t): 0 \leq t \leq \Delta t\}$, while $S^{\prime}$ associates with the set of events $\left\{\left(t^{\prime}, \vec{x}^{\prime}\right)=(0,(1 / \tilde{\gamma}) \vec{x}): \vec{x}=\overrightarrow{\tilde{v}} t\right\}$. Since the whole movement of the object as described by $S$ reduces in $S^{\prime}$ to a static situation where $t^{\prime}=0$ and since there are many $\vec{x}^{\prime}$ corresponding to the many possible values of $\vec{x}$, we conclude that relative to $S^{\prime}$ the object seems to have a spatial extension. Therefore, from the perspective of the frame $S^{\prime}$ the object cannot be seen as a particle in the classical context. This is reinforced by noticing that the possibility of considering $\vec{x}=\overrightarrow{\vec{v}} t$ leads to the vanishing of the denominator of (90) that shows that the velocity transformation law does not apply.

Let us consider now the movement of an object such that according to the frame $S^{\prime}$ the object is at rest relative to the frame $S^{\prime}$, that is, $\vec{x}^{\prime}=0$. Replacing it in (98) we now obtain $t^{\prime}=(1 / \widetilde{\gamma}) t$ and $\vec{x}=\left(c^{2} / \widetilde{v}^{2}\right) \vec{v} t$. Therefore we get

$$
\left|\frac{d \vec{x}}{d t}\right|=\frac{c^{2}}{\widetilde{v}}
$$

and with $\widetilde{v}>c$ we end up with $|d \vec{x} / d t|<c$. Here, it is natural that instead of $\overrightarrow{\vec{v}}$ we assume the relative velocity between the frames to be $\vec{V}:=\left(c^{2} / \tilde{v}^{2}\right) \overrightarrow{\tilde{v}}$, in terms of which the transformation (98) becomes

$$
\begin{gathered}
t^{\prime}=\gamma\left(t-\frac{\vec{x} \cdot \vec{V}}{V^{2}}\right), \\
\vec{x}^{\prime}=\vec{x}-(1-\gamma) \frac{\vec{x} \cdot \vec{V}}{V^{2}} \vec{V}-\gamma t \vec{V}
\end{gathered}
$$

with $\gamma=1 / \sqrt{1-\left(V^{2} / c^{2}\right)}$. Then, from a theoretical perspective, we conclude that a transformation like (98) may refer to different situations according to the interpretation one gives to $\overrightarrow{\vec{v}}$.

4.3.3. Another Superluminal Transformation. As another class of transformation let us choose $\alpha, \vec{\beta}$ such that

$$
\frac{1+\left(\beta^{2} / \alpha^{2}\right)}{1-\left(\beta^{2} / \alpha^{2}\right)}=\frac{1}{\sqrt{\left(\widetilde{v}^{2} / c^{2}\right)-1}}
$$

with $\widetilde{v}>c$, which gives

$$
\frac{\vec{\beta}}{\alpha}=-\sqrt{\frac{1-\sqrt{\left(\widetilde{v}^{2} / c^{2}\right)-1}}{1+\sqrt{\left(\widetilde{v}^{2} / c^{2}\right)-1}}} \frac{\vec{v}}{\widetilde{v}} .
$$

Replacing it in (85) we obtain the transformation

$$
\begin{gathered}
t^{\prime}=\frac{1}{\sqrt{\left(\widetilde{v}^{2} / c^{2}\right)-1}}\left\{t-\sqrt{\left.2-\frac{\widetilde{v}^{2}}{c^{2}} \frac{\vec{x} \cdot \overrightarrow{\tilde{v}}}{c \widetilde{v}}\right\}}\right. \\
\vec{x}^{\prime}=\vec{x}+\frac{1-\sqrt{\left(\widetilde{v}^{2} / c^{2}\right)-1}}{\sqrt{\left(\widetilde{v}^{2} / c^{2}\right)-1}} \frac{\vec{x} \cdot \overrightarrow{\tilde{v}}_{\vec{v}}}{\widetilde{v}^{2}}-\frac{\sqrt{2-\left(\widetilde{v}^{2} / c^{2}\right)}}{\sqrt{\left(\widetilde{v}^{2} / c^{2}\right)-1}} \frac{c}{\widetilde{v}} \overrightarrow{\vec{v}} .
\end{gathered}
$$

The condition of having real transformations requires that $\widetilde{v}<2 c$, which becomes an upper bound velocity for tachyonic motion.

4.4. Introducing the Galilei Relativity. Let us modify condition (76) writing it in the form

$$
\tau=\alpha x^{0}+\vec{\beta} \cdot \vec{x}=\alpha x^{\prime 0}-\vec{\beta} \cdot \vec{x}^{\prime},
$$

where we have introduced the absolute time $\tau$. Therefore, assuming the Galilei transformation

$$
\vec{x}^{\prime}=\vec{x}-\vec{v} \tau
$$

and using (85) and (104), we obtain that

$$
\vec{v}=-\frac{2}{1-\left(\beta^{2} / \alpha^{2}\right)} \frac{\vec{\beta}}{\alpha^{2}} .
$$


From (104) we also obtain

$$
\frac{d t}{d \tau}=\frac{1}{\alpha c+\vec{\beta} \cdot(d \vec{x} / d t)}
$$

Let us define

$$
\begin{aligned}
\vec{u}:=\frac{d \vec{x}}{d \tau}, & \overrightarrow{\vec{u}}:=\frac{d \vec{x}}{d t} \\
\vec{u}^{\prime}:=\frac{d \vec{x}^{\prime}}{d \tau}, & \overrightarrow{\vec{u}}^{\prime}:=\frac{d \vec{x}^{\prime}}{d t} .
\end{aligned}
$$

Then we obtain

$$
\vec{u}^{\prime}=\frac{\overrightarrow{\vec{u}}^{\prime}}{\alpha c-\vec{\beta} \cdot \overrightarrow{\tilde{u}}^{\prime}}, \quad \vec{u}=\frac{\overrightarrow{\vec{u}}}{\alpha c+\vec{\beta} \cdot \overrightarrow{\vec{u}}} .
$$

From the Galilei relativity we have

$$
\vec{u}^{\prime}=\vec{u}-\vec{v} .
$$

Therefore, assuming $\vec{u}^{\prime}=0$, we get $\vec{u}=\vec{v}, \overrightarrow{\widetilde{u}}=\overrightarrow{\widetilde{v}}$, and

$$
\vec{v}=\frac{\overrightarrow{\widetilde{v}}}{\alpha c+\vec{\beta} \cdot \overrightarrow{\widetilde{v}}}
$$

Replacing this last expression into (106) we end up with

$$
\overrightarrow{\widetilde{v}}=-\frac{2 c}{1+\left(\beta^{2} / \alpha^{2}\right)} \frac{\vec{\beta}}{\alpha} .
$$

In particular, we notice that

$$
\widetilde{v}<c .
$$

Then, the assumption of an absolute time and the Galilean relativity law in model II also leads to a situation where there are no superluminal frames.

The expression we obtained for $\widetilde{v}$ in (112) also leads to the same transformation of Section 4.3.1; therefore, when we incorporate the Galilei relativity into model II, we reduce the model to the standard Lorentz situation. However, different from model I, as we have seen in Section 4.3.1, we are allowed to obtain the Lorentz case without the need to impose the Galilei relativity.

4.5. The Momentum. We now search for an expression for the momentum that is conserved in collision processes. As it is well known [5], the conservation of momentum in two frames $S$ and $S^{\prime}$ brings to the analysis the transformation between them. Then, the problem here is to find an expression for the momentum that satisfies a conservation law as seen by frames related by the GLT.

Let us perform our analysis considering a particular collision process involving two particles with the same mass and such that relative to $S$ we have before the collision the particles' velocities $\overrightarrow{\tilde{u}}_{1}=\tilde{u} \hat{\eta}, \overrightarrow{\widetilde{u}}_{2}=-\tilde{u} \hat{\eta}$, and after the collision $\overrightarrow{\vec{u}}_{1}^{*}=\widetilde{u} \widehat{\eta}^{*}, \overrightarrow{\vec{u}}_{2}^{*}=-\widetilde{u} \widehat{\eta}^{*}$. (As it is typical in the analysis of collision processes, for a more general collision we would obtain constraints on the final velocities, which are not essential in order to fix the form of the function $f(\overrightarrow{\widetilde{u}})$ introduced in (116), (117).) According to $S^{\prime}$, we obtain from (90)

$$
\begin{aligned}
\overrightarrow{\vec{u}}_{1}^{\prime}= & \left(\frac{2 c}{1-\left(\beta^{2} / \alpha^{2}\right)} \frac{\vec{\beta}}{\alpha}+\widetilde{u} \vec{\omega}\right) \\
& \times\left(\frac{1+\left(\beta^{2} / \alpha^{2}\right)}{1-\left(\beta^{2} / \alpha^{2}\right)}+\widetilde{u} \frac{2}{c\left(1-\left(\beta^{2} / \alpha^{2}\right)\right)} \frac{\vec{\beta}}{\alpha} \cdot \hat{\eta}\right)^{-1}, \\
\overrightarrow{\widetilde{u}}_{2}^{\prime}= & \left(\frac{2 c}{1-\left(\beta^{2} / \alpha^{2}\right)} \frac{\vec{\beta}}{\alpha}-\tilde{u} \vec{\omega}\right) \\
& \times\left(\frac{1+\left(\beta^{2} / \alpha^{2}\right)}{1-\left(\beta^{2} / \alpha^{2}\right)}-\widetilde{u} \frac{2}{c\left(1-\left(\beta^{2} / \alpha^{2}\right)\right)} \frac{\vec{\beta}}{\alpha} \cdot \widehat{\eta}\right)^{-1}, \\
\overrightarrow{\widetilde{u}}_{1}^{\prime *}= & \left(\frac{2 c}{1-\left(\beta^{2} / \alpha^{2}\right)} \frac{\vec{\beta}}{\alpha}+\widetilde{u} \vec{\omega}^{*}\right) \\
& \times\left(\frac{1+\left(\beta^{2} / \alpha^{2}\right)}{1-\left(\beta^{2} / \alpha^{2}\right)}+\widetilde{u} \frac{2}{c\left(1-\left(\beta^{2} / \alpha^{2}\right)\right)} \frac{\vec{\beta}}{\alpha} \cdot \widehat{\eta}^{*}\right)^{-1},
\end{aligned}
$$

$$
\begin{aligned}
\overrightarrow{\vec{u}}_{2}^{\prime *}= & \left(\frac{2 c}{1-\left(\beta^{2} / \alpha^{2}\right)} \frac{\vec{\beta}}{\alpha}-\widetilde{u} \vec{\omega}^{*}\right) \\
& \times\left(\frac{1+\left(\beta^{2} / \alpha^{2}\right)}{1-\left(\beta^{2} / \alpha^{2}\right)}-\tilde{u} \frac{2}{c\left(1-\left(\beta^{2} / \alpha^{2}\right)\right)} \frac{\vec{\beta}}{\alpha} \cdot \widehat{\eta}^{*}\right)^{-1}
\end{aligned}
$$

with

$$
\begin{gathered}
\vec{\omega}:=\widehat{\eta}+\frac{2}{1-\left(\beta^{2} / \alpha^{2}\right)} \frac{\vec{\beta}}{\alpha} \cdot \widehat{\eta} \frac{\vec{\beta}}{\alpha}, \\
\vec{\omega}^{*}:=\widehat{\eta}^{*}+\frac{2}{1-\left(\beta^{2} / \alpha^{2}\right)} \frac{\vec{\beta}}{\alpha} \cdot \widehat{\eta}^{*} \frac{\vec{\beta}}{\alpha}
\end{gathered}
$$

being two vectors having arbitrary directions due to their dependence on the arbitrary unit vectors $\widehat{\eta}, \widehat{\eta}^{*}$. Following [5], we write for the momentum conservation in the frame $S$

$$
f\left(\overrightarrow{\widetilde{u}}_{1}\right) \overrightarrow{\widetilde{u}}_{1}+f\left(\overrightarrow{\widetilde{u}}_{2}\right) \overrightarrow{\widetilde{u}}_{2}=f\left(\overrightarrow{\widetilde{u}}_{1}^{*}\right) \overrightarrow{\widetilde{u}}_{1}^{*}+f\left(\overrightarrow{\widetilde{u}}_{2}^{*}\right) \overrightarrow{\widetilde{u}}_{2}^{*}
$$

and relative to $S^{\prime}$ it becomes (assuming that the form of the function $f$ is invariant)

$$
f\left(\overrightarrow{\widetilde{u}}_{1}^{\prime}\right) \overrightarrow{\widetilde{u}}_{1}^{\prime}+f\left(\overrightarrow{\widetilde{u}}_{2}^{\prime}\right) \overrightarrow{\widetilde{u}}_{2}^{\prime}=f\left(\overrightarrow{\widetilde{u}}_{1}^{\prime *}\right) \overrightarrow{\widetilde{u}}_{1}^{\prime *}+f\left(\overrightarrow{\widetilde{u}}_{2}^{\prime *}\right) \overrightarrow{\widetilde{u}}_{2}^{\prime *}
$$


that is equivalent to

$$
\begin{aligned}
& \tilde{u} \vec{\omega}\left\{\left(f\left(\overrightarrow{\widetilde{u}}_{1}^{\prime}\right)\right)\right. \\
& \times\left(\frac{1+\left(\beta^{2} / \alpha^{2}\right)}{1-\left(\beta^{2} / \alpha^{2}\right)}+\widetilde{u} \frac{2}{c\left(1-\left(\beta^{2} / \alpha^{2}\right)\right)} \frac{\vec{\beta}}{\alpha} \cdot \hat{\eta}\right)^{-1} \\
& -\left(f\left(\overrightarrow{\widetilde{u}}_{2}^{\prime}\right)\right) \\
& \left.\times\left(\frac{1+\left(\beta^{2} / \alpha^{2}\right)}{1-\left(\beta^{2} / \alpha^{2}\right)}-\widetilde{u} \frac{2}{c\left(1-\left(\beta^{2} / \alpha^{2}\right)\right)} \frac{\vec{\beta}}{\alpha} \cdot \widehat{\eta}\right)^{-1}\right\} \\
& -\widetilde{u} \vec{\omega}^{*}\left\{\left(f\left(\overrightarrow{\widetilde{u}}_{1}^{\prime *}\right)\right)\right. \\
& \times\left(\frac{1+\left(\beta^{2} / \alpha^{2}\right)}{1-\left(\beta^{2} / \alpha^{2}\right)}+\tilde{u} \frac{2}{c\left(1-\left(\beta^{2} / \alpha^{2}\right)\right)} \frac{\vec{\beta}}{\alpha} \cdot \hat{\eta}^{*}\right)^{-1} \\
& -\left(f\left(\overrightarrow{\vec{u}}_{2}^{\prime *}\right)\right) \\
& \times\left(\frac{1+\left(\beta^{2} / \alpha^{2}\right)}{1-\left(\beta^{2} / \alpha^{2}\right)}\right. \\
& \left.\left.-\tilde{u} \frac{2}{c\left(1-\left(\beta^{2} / \alpha^{2}\right)\right)} \frac{\vec{\beta}}{\alpha} \cdot \widehat{\eta}^{*}\right)^{-1}\right\}
\end{aligned}
$$$$
+\frac{\vec{\beta}}{\alpha} \frac{2 c}{1-\left(\beta^{2} / \alpha^{2}\right)}
$$$$
\times\left\{\left(f\left(\overrightarrow{\widetilde{u}}_{1}^{\prime}\right)\right)\right.
$$$$
\times\left(\frac{1+\left(\beta^{2} / \alpha^{2}\right)}{1-\left(\beta^{2} / \alpha^{2}\right)}+\widetilde{u} \frac{2}{c\left(1-\left(\beta^{2} / \alpha^{2}\right)\right)} \frac{\vec{\beta}}{\alpha} \cdot \widehat{\eta}\right)^{-1}
$$$$
+\left(f\left(\overrightarrow{\tilde{u}}_{2}^{\prime}\right)\right)
$$$$
\times\left(\frac{1+\left(\beta^{2} / \alpha^{2}\right)}{1-\left(\beta^{2} / \alpha^{2}\right)}-\tilde{u} \frac{2}{c\left(1-\left(\beta^{2} / \alpha^{2}\right)\right)} \frac{\vec{\beta}}{\alpha} \cdot \widehat{\eta}\right)^{-1}
$$$$
-\left(f\left(\overrightarrow{\vec{u}}_{1}^{\prime *}\right)\right)
$$$$
\times\left(\frac{1+\left(\beta^{2} / \alpha^{2}\right)}{1-\left(\beta^{2} / \alpha^{2}\right)}+\widetilde{u} \frac{2}{c\left(1-\left(\beta^{2} / \alpha^{2}\right)\right)} \frac{\vec{\beta}}{\alpha} \cdot \widehat{\eta}^{*}\right)^{-1}
$$$$
-\left(f\left(\overrightarrow{\widetilde{u}}_{2}^{\prime *}\right)\right)
$$

$$
\left.\begin{array}{l}
\times\left(\frac{1+\left(\beta^{2} / \alpha^{2}\right)}{1-\left(\beta^{2} / \alpha^{2}\right)}\right. \\
\left.\quad-\tilde{u} \frac{2}{c\left(1-\left(\beta^{2} / \alpha^{2}\right)\right)} \frac{\vec{\beta}}{\alpha} \cdot \widehat{\eta}^{*}\right)^{-1}
\end{array}\right\}
$$$$
=0 \text {. }
$$

From the arbitrariness of the scattering directions $\widehat{\eta}, \widehat{\eta}^{*}$, we must have

$$
\begin{aligned}
\left(f\left(\overrightarrow{\vec{u}}_{1}^{\prime}\right)\right)\left(\frac{1+\left(\beta^{2} / \alpha^{2}\right)}{1-\left(\beta^{2} / \alpha^{2}\right)}+\tilde{u} \frac{2}{c\left(1-\left(\beta^{2} / \alpha^{2}\right)\right)} \frac{\vec{\beta}}{\alpha} \cdot \widehat{\eta}\right)^{-1} \\
=\left(f\left(\overrightarrow{\vec{u}}_{2}^{\prime}\right)\right) \\
\quad \times\left(\frac{1+\left(\beta^{2} / \alpha^{2}\right)}{1-\left(\beta^{2} / \alpha^{2}\right)}-\tilde{u} \frac{2}{c\left(1-\left(\beta^{2} / \alpha^{2}\right)\right)} \frac{\vec{\beta}}{\alpha} \cdot \hat{\eta}\right)^{-1} \\
=\left(f\left(\overrightarrow{\tilde{u}}_{1}^{\prime *}\right)\right) \\
\quad \times\left(\frac{1+\left(\beta^{2} / \alpha^{2}\right)}{1-\left(\beta^{2} / \alpha^{2}\right)}+\widetilde{u} \frac{2}{c\left(1-\left(\beta^{2} / \alpha^{2}\right)\right)} \frac{\vec{\beta}}{\alpha} \cdot \hat{\eta}^{*}\right)^{-1} \\
=\left(f\left(\overrightarrow{\vec{u}}_{2}^{\prime *}\right)\right) \\
\quad \times\left(\frac{1+\left(\beta^{2} / \alpha^{2}\right)}{1-\left(\beta^{2} / \alpha^{2}\right)}-\tilde{u} \frac{2}{c\left(1-\left(\beta^{2} / \alpha^{2}\right)\right)} \frac{\vec{\beta}}{\alpha} \cdot \widehat{\eta}^{*}\right)^{-1} .
\end{aligned}
$$

In order to satisfy this condition we notice that from (91) we obtain

$$
\begin{aligned}
\sqrt{\mid 1}-\frac{\tilde{u}^{\prime 2}}{c^{2}} \mid \\
=\left(\sqrt{\left|1-\left(\frac{\tilde{u}^{2}}{c^{2}}\right)\right|}\right) \\
\quad \times\left(\left|\frac{1+\left(\beta^{2} / \alpha^{2}\right)}{1-\left(\beta^{2} / \alpha^{2}\right)}+\frac{2}{1-\left(\beta^{2} / \alpha^{2}\right)} \frac{\vec{\beta}}{\alpha} \cdot \frac{\overrightarrow{\tilde{u}}}{c}\right|\right)^{-1} .
\end{aligned}
$$

For both cases we analyzed in Sections 4.3.1, 4.3.2, we observe that $\beta / \alpha<1$ and for the case of a subluminal particle, that is, $u<c, u^{\prime}<c$, we obtain

$$
\frac{1+\left(\beta^{2} / \alpha^{2}\right)}{1-\left(\beta^{2} / \alpha^{2}\right)}+\frac{2}{1-\left(\beta^{2} / \alpha^{2}\right)} \frac{\vec{\beta}}{\alpha} \cdot \frac{\overrightarrow{\tilde{u}}}{c}>0
$$

which suggest us to take for $f(\widetilde{u})$ the following expression:

$$
f(\widetilde{u})=\frac{k}{\sqrt{1-\left(\tilde{u}^{2} / c^{2}\right)}} .
$$


In the case of a superluminal particle, that is, $u>c, u^{\prime}>c$, we have in general

$$
\frac{1+\left(\beta^{2} / \alpha^{2}\right)}{1-\left(\beta^{2} / \alpha^{2}\right)}+\frac{2}{1-\left(\beta^{2} / \alpha^{2}\right)} \frac{\vec{\beta}}{\alpha} \cdot \frac{\overrightarrow{\tilde{u}}}{c}<0
$$

which suggest us to take

$$
f(\widetilde{u})=\frac{k \operatorname{sgn}\left[1+\left(\beta^{2} / \alpha^{2}\right)+2(\vec{\beta} / \alpha) \cdot(\overrightarrow{\tilde{u}} / c)\right]}{\sqrt{\left|1-\left(\widetilde{u}^{2} / c^{2}\right)\right|}} .
$$

The constant $k$ is identified with the rest mass of the particle in the case that one can effectively define such frame. In the case of tachyons we refer to the discussion of [13]. The term in the numerator, $\operatorname{sgn}\left[1+\left(\beta^{2} / \alpha^{2}\right)+2(\vec{\beta} / \alpha) \cdot(\overrightarrow{\vec{u}} / c)\right]$, becomes necessary in order to fulfill the conservation of momentum as expressed in condition (119). This term introduces a dependence of the tachyon mass with the quantity $\vec{\beta} / \alpha$, which is ultimately a parameter depending on the relative velocity between the frames. A detailed discussion on the implications of this term may be found in [13].

\section{An Alternative Way to Induce a Concept of Absolute Time}

The concept of the absolute time was introduced in models I and II by means of certain relations involving the space and time variables, (5), (104). These relations may be seen as kinematical requirements allowing to bring the special and the Galilei relativity into the models. In this view, we could think on the physical and the absolute time without having to consider them as coordinates of a 4-dimensional space. Now, we reverse the construction and we will show that by considering a higher dimensional space, we can think on the absolute time with no reference to kinematical relations.

5.1. Setting the Lorentz Transformation in $\mathbb{R}^{(1,4)}$. We denote by $\mathbb{R}^{(1,4)}$ the space $\mathbb{R}^{5}$ endowed with the quadratic form

$$
Q\left(x^{a}\right)=-\left(x^{0}\right)^{2}+\left(x^{1}\right)^{2}+\left(x^{2}\right)^{2}+\left(x^{3}\right)^{2}+\left(x^{4}\right)^{2} .
$$

Here $\mathbb{R}^{(1,4)} \ni x \equiv\left(x^{a}\right), a=0,1,2,3,4$. The transformations leaving $Q$ invariant have the form

$$
x^{\prime a}:=\Lambda_{b}^{a} x^{b}
$$

with $\left[\Lambda_{b}^{a}\right] \in S O(1,4)$. We consider now two subgroups of $S O(1,4)$ as follows. The first consists of transformations defined by matrices

$$
L_{\Lambda}:=\left[\begin{array}{cc}
\Lambda_{v}^{\mu} & \mathbf{0} \\
\mathbf{0} & 1
\end{array}\right]
$$

with $\Lambda \in \operatorname{SO}(1,3)(\mu, v=0,1,2,3)$. The second consists on transformations defined by matrices

$$
L_{\mathscr{R}}:=\left[\begin{array}{cc}
1 & \mathbf{0} \\
\mathbf{0} & \mathscr{R}_{j}^{i}
\end{array}\right]
$$

with $\mathscr{R} \in S O(4)(i, j=1,2,3,4)$.
Now, we define new coordinates as follows.

(i) $\left(x^{a} / x^{4}\right)=\left(\widehat{x}^{\mu}, 1\right)$ with $\widehat{x}^{\mu} \equiv x^{\mu} / x^{4}$. For the subgroup $\left\{L_{\Lambda}\right\} \subset S O(1,4)$ the transformation (126) becomes

$$
x^{\prime \mu}=\Lambda_{v}^{\mu} x^{\nu} \quad x^{\prime 4}=x^{4}
$$

and in particular

$$
\widehat{x}^{\prime \mu}=\Lambda_{v}^{\mu} \widehat{x}^{\nu}
$$

(ii) $\left(x^{a} / x^{0}\right)=\left(1, \tilde{x}^{i}\right)$, with $\tilde{x}^{i} \equiv x^{i} / x^{0}$. For the subgroup $\left\{L_{\mathscr{R}}\right\} \subset S O(1,4)$ the transformation (126) becomes

$$
x^{\prime 0}=x^{0}, \quad x^{\prime i}=\mathscr{R}_{j}^{i} x^{j}
$$

and in particular

$$
\tilde{x}^{i}=\mathscr{R}_{j}^{i} \tilde{x}^{j}
$$

We notice that these coordinates $\widehat{x} \equiv\left(\widehat{x}^{\mu}\right)$ and $\tilde{x} \equiv\left(\tilde{x}^{i}\right)$ cannot be identified with the coordinates of the hyperplanes $x^{0}=0, x^{4}=0$ of $\mathbb{R}^{(1,4)}$.

5.2. Inducing Lorentz Transformation in Euclidean Space $\mathbb{R}^{4}$. There is a natural way to reinterpret the coordinates $\widehat{x}=$ $\left(\widehat{x}^{\mu}\right), \tilde{x}=\left(\tilde{x}^{i}\right)$ as local coordinates on certain open sets of the real projective space $\mathbb{R} \mathrm{P}^{4}[14]$. In fact, let $\mathbb{R} \mathrm{P}^{4} \ni$ $\left[x^{0}, x^{1}, x^{2}, x^{3}, x^{4}\right]:=\left\{\lambda\left(x^{0}, x^{1}, x^{2}, x^{3}, x^{4}\right): \lambda \in \mathbb{R}^{*}\right\}$. Recall that a smooth atlas for $\mathbb{R P} \mathrm{P}^{4}$ is given by $\left\{\left(V_{a}, \varphi_{a}\right)\right\}_{a=0, \ldots, 4}$, where $V_{a}:=\left\{\left[x^{0}, x^{1}, x^{2}, x^{3}, x^{4}\right]: x^{a} \neq 0\right\}$ and

$$
\begin{gathered}
\varphi_{a}: V_{a} \longrightarrow \mathbb{R}^{4} \\
{[x] \equiv\left[x^{0}, x^{1}, x^{2}, x^{3}, x^{4}\right]} \\
\longrightarrow \varphi_{a}\left(\left[x^{0}, x^{1}, x^{2}, x^{3}, x^{4}\right]\right) \\
:=\left(\frac{x^{0}}{x^{a}}, \ldots, \frac{x^{a-1}}{x^{a}}, \frac{x^{a+1}}{x^{a}}, \ldots, \frac{x^{4}}{x^{a}}\right)
\end{gathered}
$$

which shows that $\hat{x}=\varphi_{4}([x])$ and $\tilde{x}=\varphi_{0}([x])$. (Notice that the space $\mathbb{R}^{4}$, appearing as the image of the homeomorphisms $\varphi_{a}$, is not seen as a subset of $\mathbb{R}^{(1,4)}$.)

The transformation on $\mathbb{R}^{(1,4)}, x^{\prime a}=\Lambda_{b}^{a} x^{b}$, induces a transformation on $\mathbb{R P}^{4}:[x] \rightarrow[\Lambda x]$ that has the particular cases

$$
\begin{gathered}
{\left[x^{0}, x^{1}, x^{2}, x^{3}, x^{4}\right] \stackrel{L_{\Lambda}}{\longrightarrow}\left[\Lambda_{\alpha}^{0} x^{\alpha}, \Lambda_{\alpha}^{1} x^{\alpha}, \Lambda_{\alpha}^{2} x^{\alpha}, \Lambda_{\alpha}^{3} x^{\alpha}, x^{4}\right],} \\
{\left[x^{0}, x^{1}, x^{2}, x^{3}, x^{4}\right] \stackrel{L_{\mathscr{R}}}{\longrightarrow}\left[x^{0}, \mathscr{R}_{i}^{1} x^{i}, \mathscr{R}_{i}^{2} x^{i}, \mathscr{R}_{i}^{3} x^{i}, \mathscr{R}_{i}^{4} x^{i}\right] .}
\end{gathered}
$$

Therefore, $\varphi_{4} \circ L_{\Lambda} \circ \varphi_{4}^{-1}$ reduces to an ordinary Lorentz transformation in $\varphi_{4}\left(V_{4}\right) \simeq \mathbb{R}^{4}: \widehat{x}^{\mu} \rightarrow \widehat{x}^{\prime \mu}=\Lambda_{\nu}^{\mu} \widehat{x}^{\nu}$, while $\varphi_{0} \circ L_{\mathscr{R}} \circ \varphi_{0}^{-1}$ reduces to a 4 -dimensional rotation in $\varphi_{0}\left(V_{0}\right) \simeq \mathbb{R}^{4}: \tilde{x}^{i} \rightarrow \tilde{x}^{i}=\mathscr{R}_{j}^{i} \tilde{x}^{j}$. We have then modelled 
Minkowski and Euclidean four-space as homeomorphic to open sets $\varphi_{4}\left(V_{4}\right), \varphi_{0}\left(V_{0}\right)$ of projective space, where Lorentz and 4-dimensional rotations are induced by transformations (129), (131) of $S O(1,4)$ acting in $\mathbb{R}^{5}$. In the intersection of the neighborhoods, $V_{0} \cap V_{4}$, we have $\tilde{x}^{i}=\varphi_{0} \circ \varphi_{4}^{-1}(\widehat{x})$, that is,

$$
\tilde{x}^{1}=\frac{\widehat{x}^{1}}{\widehat{x}^{0}}, \quad \tilde{x}^{2}=\frac{\widehat{x}^{2}}{\widehat{x}^{0}}, \quad \tilde{x}^{3}=\frac{\widehat{x}^{3}}{\widehat{x}^{0}}, \quad \tilde{x}^{4}=\frac{1}{\widehat{x}^{0}} .
$$

Therefore, in the intersection $V_{0} \cap V_{4}$ an ordinary Lorentz transformation on $\varphi_{4}\left(V_{4}\right), \widehat{x}^{\mu} \rightarrow \hat{x}^{\prime \mu}=\Lambda^{\mu}{ }_{\nu} \hat{x}^{\nu}$, induces on $\varphi_{0}\left(V_{0}\right)$ the transformation

$$
\tilde{x}^{i} \longrightarrow \tilde{x}^{\prime i}=\frac{L_{\Lambda 0}^{i}+L_{\Lambda j}^{i} \tilde{x}^{j}}{L_{\Lambda 0}^{0}+L_{\Lambda j}^{0} \tilde{x}^{j}}
$$

that also obeys a group law, for example,

$$
\begin{aligned}
& \widehat{x} \stackrel{\Lambda_{(1)}}{\longrightarrow} \widehat{x}_{(1)} \stackrel{\Lambda_{(2)}}{\longrightarrow} \widehat{x}_{(2)} \\
& \tilde{x} \stackrel{L_{(1)}}{\longrightarrow} \tilde{x}_{(1)} \stackrel{L_{(2)}}{\longrightarrow} \tilde{x}_{(2)}
\end{aligned}
$$

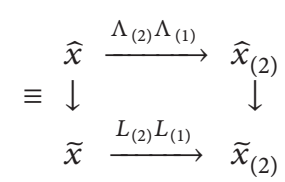

That is,

$$
\tilde{x}_{(2)}^{i}=\frac{\left(L_{(2)} L_{(1)}\right)_{0}^{i}+\left(L_{(2)} L_{(1)}\right)_{j}^{i} \tilde{x}^{j}}{\left(L_{(2)} L_{(1)}\right)_{0}^{0}+\left(L_{(2)} L_{(1)}\right)_{j}^{0} \tilde{x}^{j}}
$$

Equation (136) may be seen as a nonlinear realization of the Lorentz group in $\varphi_{0}\left(V_{0}\right)$.

\subsection{Interpreting the Boost Transformation in Euclidean Space}

$\mathbb{R}^{4}$. Now, let us introduce some physics in our analysis. So far, we have considered homogeneous coordinates $\widehat{x}, \tilde{x}$ that carry no dimension. In order to give a physical meaning to them let us assume that there is a scale factor $\lambda$ with dimension of length and let us define $y^{i}:=\lambda \widetilde{x}^{i}, y^{\prime i}:=\lambda^{\prime} \tilde{x}^{\prime i}$. Here $\lambda^{\prime}$ is the transformed scale factor whose form is left arbitrary for a moment. Then, transformation (136) may be seen as a transformation

$$
y^{i} \longrightarrow y^{\prime i}=\lambda^{\prime} \frac{L_{\Lambda 0}^{i} \lambda+L_{\Lambda j}^{i} y^{j}}{L_{\Lambda 0}^{0} \lambda+L_{\Lambda j}^{0} y^{j}} .
$$

For a boost we have $\Lambda_{0}^{0}=\gamma, \quad \Lambda_{\bar{i}}^{0}=\Lambda_{0}^{\bar{i}}=-(\gamma / c) v^{\bar{i}}, \quad \Lambda_{\bar{j}}^{\bar{i}}=$ $\delta_{\bar{j}}^{\bar{i}}-(1-\gamma)\left(v^{\bar{i}} v^{\bar{j}} / v^{2}\right)$, and we obtain

$$
\begin{gathered}
y^{\prime \bar{i}}=\lambda^{\prime} \frac{y^{\bar{i}}-(1-\gamma)\left(y^{\bar{j}} v^{\bar{j}} / v^{2}\right) v^{\bar{i}}-(\gamma / c) \lambda v^{\bar{i}}}{\gamma\left(\lambda-(1 / c) y^{\bar{j}} v^{\bar{j}}\right)}, \\
y^{\prime 4}=\lambda^{\prime} \frac{y^{4}}{\gamma\left(\lambda-(1 / c) y^{\bar{j}} v^{\bar{j}}\right)},
\end{gathered}
$$

where $\bar{i}=1,2,3$. Now, let us assume that the scale factor writes as $\lambda^{\prime}=c t^{\prime}, \lambda=c t$ with $c$ being the speed of light and $t$ and $t^{\prime}$ having the dimension of time. Here, denoting $\vec{y}=\left(y^{\bar{i}}\right), \vec{v}=\left(v^{\bar{i}}\right)(140)$ becomes

$$
\begin{gathered}
\vec{y}^{\prime}=t^{\prime} \frac{\vec{y}-(1-\gamma)\left((\vec{y} \cdot \vec{v}) / v^{2}\right) \vec{v}-\gamma t \vec{v}}{\gamma\left(t-\left((\vec{y} \cdot \vec{v}) / c^{2}\right)\right)}, \\
y^{\prime 4}=t^{\prime} \frac{y^{4}}{\gamma\left(t-\left((\vec{y} \cdot \vec{v}) / c^{2}\right)\right)} .
\end{gathered}
$$

If $t^{\prime}=\gamma\left(t-\left((\vec{y} \cdot \vec{v}) / c^{2}\right)\right)$, we obtain

$$
\vec{y}^{\prime}=\vec{y}-(1-\gamma) \frac{\vec{y} \cdot \vec{v}}{v^{2}} \vec{v}-\gamma t \vec{v},
$$

$$
y^{\prime 4}=y^{4}
$$

Equation (142) together with $t^{\prime}=\gamma\left(t-\left((\vec{y} \cdot \vec{v}) / c^{2}\right)\right)$ constitutes the standard transformation of special relativity, and $y^{\prime 4}=$ $y^{4}$ can be considered as defining the absolute time, for example, $\tau \equiv y^{\prime 4} / c=y^{4} / c$. In this way, the absolute time acquires an intrinsic characteristic as the coordinate (in fact with $\left.y^{4} / c\right)$ of a 4 -dimensional Euclidean space $\mathbb{R}^{4} \simeq \varphi_{0}\left(V_{0}\right)$, while the physical time of SR appears as a noninvariant scale factor (through the relation $\lambda=c t, \lambda^{\prime}=c t^{\prime}$ ) transforming homogeneous coordinates $\tilde{x}^{i}$ of Euclidean space $\mathbb{R}^{4}$ into dimensional coordinates $y^{i}$. If in addition we impose the kinematical relation of the Galilei relativity, $\vec{y}^{\prime}=\vec{y}-\vec{v} \tau$, we end up with $\tau=(1-\gamma)\left((\vec{y} \cdot \vec{v}) / v^{2}\right) \vec{v}+\gamma \vec{v} t$.

\section{Conclusion}

In our work we have consistently unified the Galilei and the Special relativities. In model I the main equations were $\vec{x}^{\prime}=$ $\vec{x}-\vec{v} \tau$ and $\vec{x}^{\prime 2}-c^{2} t^{\prime 2}=\vec{x}^{2}-c^{2} t^{2}$, while in model II we have used $\vec{x}^{\prime}=\vec{x}-\vec{v} \tau$ and $\tau=\alpha x^{0}+\vec{\beta} \cdot \vec{x}=\alpha x^{\prime 0}-\vec{\beta} \cdot \vec{x}^{\prime}$. Here, contrarily to the intrinsic geometric meaning associated with the relation $\vec{x}^{\prime 2}-c^{2} t^{\prime 2}=\vec{x}^{2}-c^{2} t^{2}$, the role played by $\alpha x^{0}+\vec{\beta} \cdot \vec{x}=\alpha x^{\prime 0}-\vec{\beta} \cdot \vec{x}^{\prime}$ is not clear. If there is a certain anisotropy in space, represented by a particular direction in space (not necessarily related to the relative velocity of the two frames), then we could encode it into $\vec{\beta}$. In this way, the physics derived from the general transformation (85) may provide a framework to describe situations other than the one we contemplated in our work.

The assumption of the absolute time leads to a distinction between the velocities $\vec{v}=d \vec{x} / d \tau$ and $\overrightarrow{\widetilde{v}}=d \overrightarrow{\widetilde{x}} / d t$. As we have seen in (32), (113), the Galilei relativity imposes a relation between these velocities in such way that $\widetilde{v}$ becomes less than $c$, which rules out tachyons since in SR we consider velocities only as derivatives with respect to $t$. However, as we have seen in model II, it is possible to have superluminal frames provided we do not impose the existence of the absolute time and the Galilei relativity. Having distinguished between the absolute and the physical time, the assumption of the constancy of the speed of light is understood only with 
respect to the derivative relative to the physical time. In fact, as we have seen in the discussion of Section 3.4.2, for the light speed calculated as a derivative relative to the absolute time, we obtained $c_{S} \neq c_{S^{\prime}}$.

Finally, the implications of the existence of an absolute time in the formulation of a quantum theory are an old topic. Dirac, for example, has used it in his attempt to develop electrodynamics [15]. In the context of our work, one possibility that arises is related to the development of quantum theory following the Schwinger Quantum Action Principle (SQAP). When applied to quantum mechanics this approach uses an hamiltonian formalism that singles out the time as parameter. As we have shown in [16], a consequence of the application of a modified form of the SQAP to quantum mechanics has lead to the commutator of the Lie algebra of the Galilei group. Now, we could apply the same procedure using the absolute time as parameter together with its relation to the physical time as given by (76) and search for the corresponding modifications that would originate in the Galilei algebra. Then, we could check if it corresponds to the group of transformation leaving the quantity $\tau=\alpha x^{0}+\vec{\beta} \cdot \vec{x}=$ $\alpha x^{\prime 0}-\vec{\beta} \cdot \vec{x}^{\prime}$ invariant.

\section{Acknowledgments}

The authors thank all the staff of the library of Centro Brasileiro de Pesquisas Físicas (CBPF), in particular Vera, Fátima, Edileuza, Rosa, and Ramalho. Marcelo Carvalho thanks Aurelina Carvalho, José E. Carvalho, Aureliana Cabral Raposo, and the enlightened conversations with Yohan Ricardo. Alexandre Lyra thanks Eliana N. Lyra de Oliveira for the permanent support and Maria I. Lyra (in memoriam) and dedicates this work (in memoriam) to his sister Anita Lyra de Oliveira with Down syndrome, recently deceased. Both authors thank professor A. F. da F. Teixeira of CBPF for some comments on part of work. This work was done in honour of $\overline{\mathrm{IC}} \overline{\mathrm{XC}}, \overline{\mathrm{MP}} \overline{\Theta \Upsilon}$.

\section{References}

[1] O. Sela, B. Tamir, S. Dolev, and A. C. Elitzur, "Can special relativity be derived from Galilean mechanics alone?" Foundations of Physics, vol. 39, no. 5, pp. 499-509, 2009.

[2] R. T. Cahill, "Unravelling Lorentz covariance and the spacetime formalism," Progress in Physics, vol. 4, pp. 19-24, 2008.

[3] E. A. Desloge and R. J. Philpott, "Uniformly accelerated reference frames in special relativity," American Journal of Physics, vol. 55, no. 3, pp. 252-261, 1987.

[4] A. P. French, Newtonian Mechanics, W.W. Norton, New York, NY, USA, 1971.

[5] C. Møller, The Theory of Relativity, Oxford University Press, Oxford, UK, 1972.

[6] E. A. Desloge, Classical Mechanics. Vol. 1, John Wiley \& Sons, New York, NY, USA, 1986.

[7] L. Landau and E. Lifshitz, The Classical Theory of Fields, Butterworth-Heinemann, 1980.

[8] A. Einstein, Relativity, Three Rivers Press, New York, NY, USA, 1961.
[9] T. S. Shankara, "Tachyons via supersonics," Foundations of Physics, vol. 4, no. 1, pp. 97-104, 1974.

[10] G. H. Duffey, "Tachyons and superluminal wave groups," Foundations of Physics, vol. 5, no. 2, pp. 349-354, 1975.

[11] V. I. Ritus, “On the difference between Wigner's and Møller's approaches to the description of Thomas precession," PhysicsUspekhi, vol. 50, no. 1, pp. 95-101, 2007.

[12] E. Kapuscik, "Special theory of relativity without special assumptions and tachyonic motion," Condensed Matter Physics, vol. 13, no. 4, Article ID 43102, 8 pages, 2010.

[13] M. I. Park and Y. J. Park, "On the foundation of the relativistic dynamics with the tachyon," Il Nuovo Cimento B, vol. 111, no. 11, pp. 1333-1368, 1996.

[14] M. P. do Carmo, Geometria Riemaniana, IMPA, Projeto Euclides, Rio de Janeiro, Brazil, 1988.

[15] P. A. M. Dirac, "The Lorentz transformation and absolute time," Physica, vol. 19, pp. 888-896, 1953.

[16] M. Carvalho and A. Lyra, "The quantum action principle revisited," Canadian Journal of Physics, vol. 86, no. 5, pp. 703$712,2008$. 


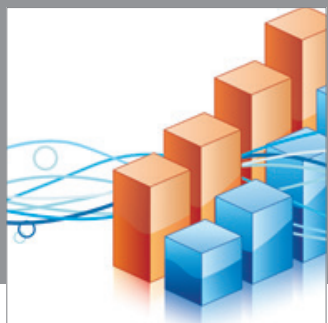

Advances in

Operations Research

mansans

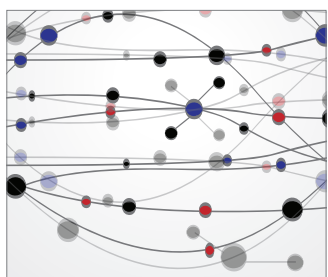

The Scientific World Journal
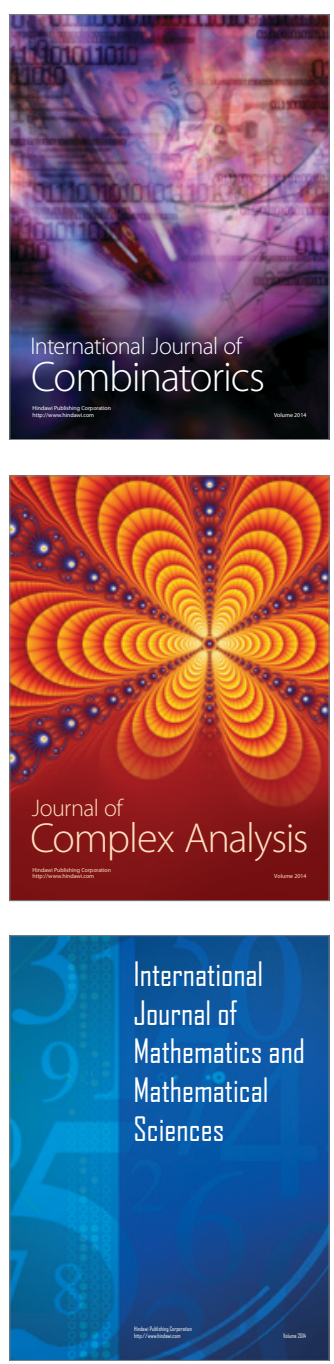
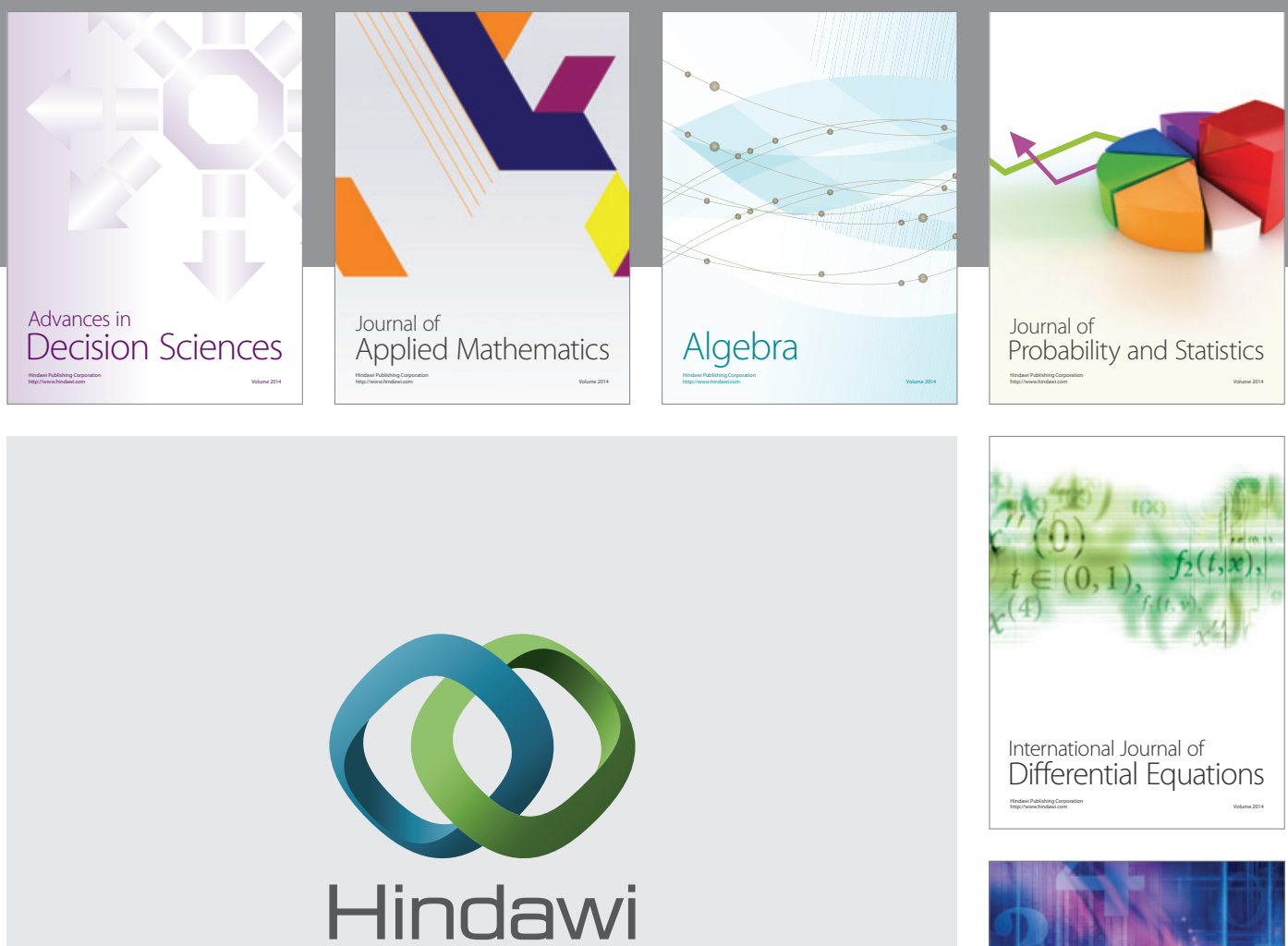

Submit your manuscripts at http://www.hindawi.com
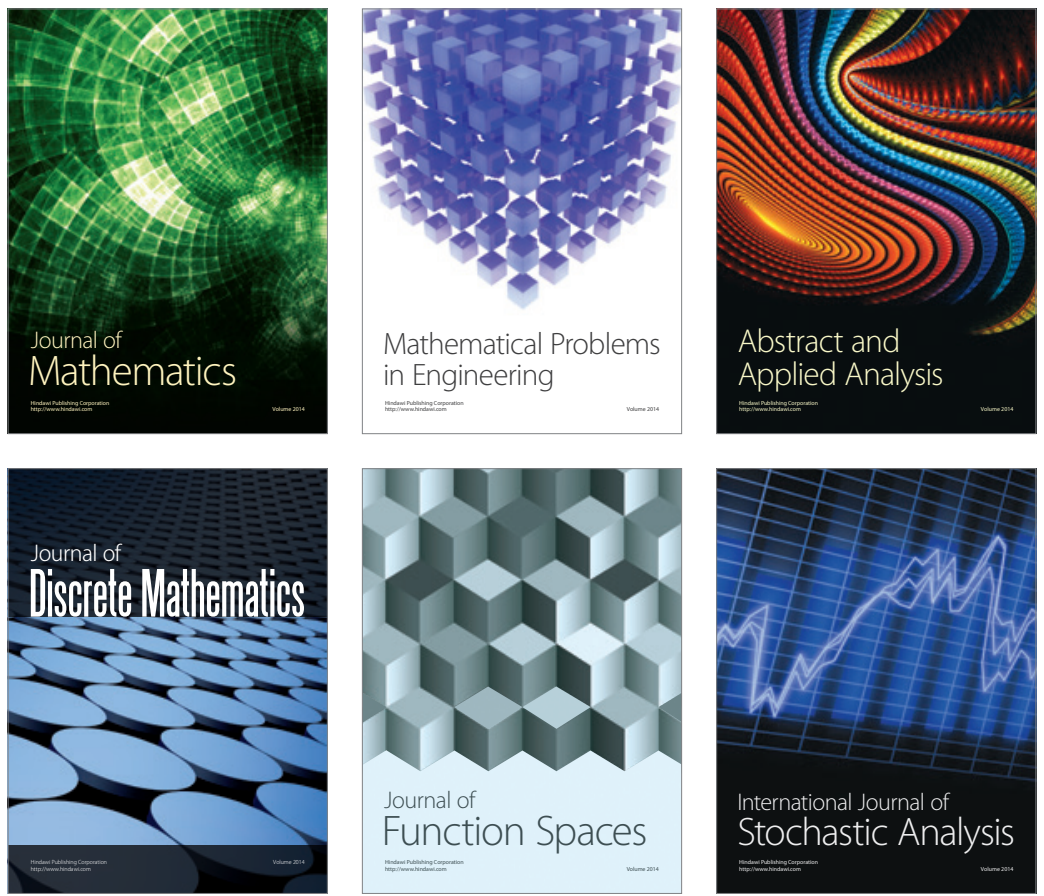

Journal of

Function Spaces

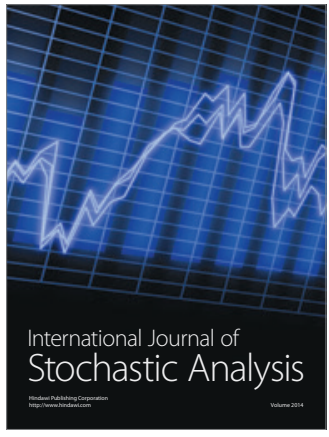

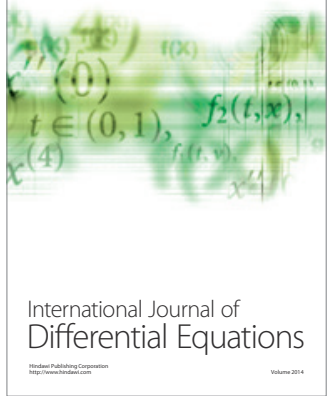
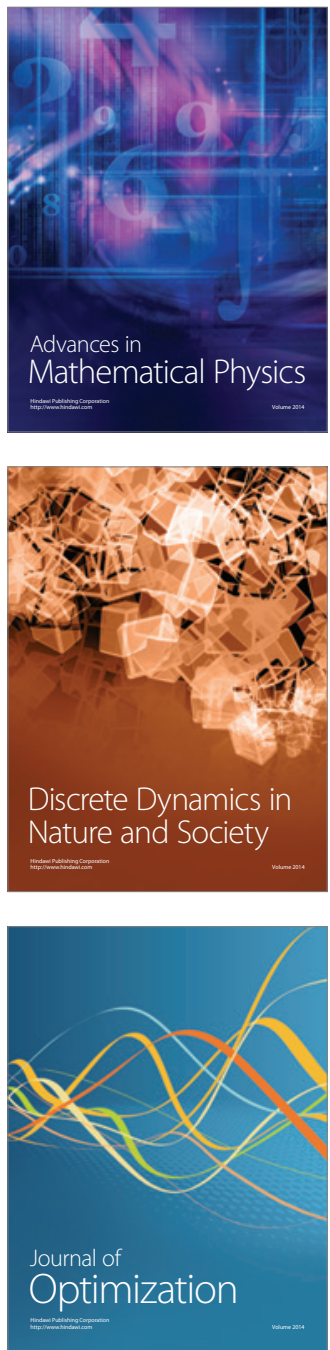